\title{
Metric for attractor overlap
}

\section{Rishabh Ishar ${ }^{1} \dagger \ddagger$ Eurika Kaiser ${ }^{2}$, Marek Morzynski ${ }^{3}$, Daniel Fernex ${ }^{4}$, Richard Semaan ${ }^{4}$, Marian Albers ${ }^{5}$, Pascal S. Meysonnat ${ }^{5}$, Wolfgang Schröder ${ }^{5,6}$, and Bernd R. Noack ${ }^{7,4,8,9}$}

\author{
${ }^{1}$ Department of Mechanical Engineering, Punjab Engineering College, Chandigarh 160012, \\ India \\ ${ }^{2}$ University of Washington, Department of Mechanical Engineering, Stevens Way, Box 352600, \\ Seattle, WA 98195, United States \\ ${ }^{3}$ Poznań University of Technology, Chair of Virtual Engineering, Jana Pawla II 24, PL 60-965 \\ Poznań, Poland \\ ${ }^{4}$ Institut für Strömungsmechanik, Technische Universität Braunschweig, Hermann-Blenk-Str. \\ 37, 38108 Braunschweig, Germany \\ ${ }^{5}$ Institute of Aerodynamics, RWTH Aachen University, Wüllnerstr. 5a, 52062 Aachen, \\ Germany \\ ${ }^{6}$ Forschungszentrum Jülich, JARA-High-Performance Computing, 52425 Jülich, Germany \\ ${ }^{7}$ Laboratoire d'Informatique pour la Mécanique et les Sciences de l'Ingénieur, LIMSI-CNRS, \\ Rue John von Neumann, Campus Universitaire d'Orsay, Bât 508, 91403 Orsay Cedex, France \\ ${ }^{8}$ Institut für Strömungsmechanik und Technische Akustik (ISTA), Technische Universität \\ Berlin, Müller-Breslau-Straße 8, 10623 Berlin, Germany \\ ${ }^{9}$ Institute for Turbulence-Noise-Vibration Interaction and Control, Harbin Institute of \\ Technology, Shenzhen Campus, China
}

(Received xx; revised xx; accepted $\mathrm{xx}$ )

We present the first general metric for attractor overlap (MAO) facilitating an unsupervised comparison of flow data sets. The starting point is two or more attractors, i.e., ensembles of states representing different operating conditions. The proposed metric generalizes the standard Hilbert-space distance between two snapshots to snapshot ensembles of two attractors. A reduced-order analysis for big data and many attractors is enabled by coarse-graining the snapshots into representative clusters with corresponding centroids and population probabilities. For a large number of attractors, MAO is augmented by proximity maps for the snapshots, the centroids, and the attractors, giving scientifically interpretable visual access to the closeness of the states. The coherent structures belonging to the overlap and disjoint states between these attractors are distilled by few representative centroids. We employ MAO for two quite different actuated flow configurations: a two-dimensional wake with vortices in a narrow frequency range and three-dimensional wall turbulence with broadband spectrum. In the first application, seven control laws are applied to the fluidic pinball, i.e., the two-dimensional flow around three circular cylinders whose centers form an equilateral triangle pointing in the upstream direction. These seven operating conditions comprise unforced shedding, boat tailing, base bleed, high- and low-frequency forcing as well as two opposing Magnus effects. In the second example, MAO is applied to three-dimensional simulation data from an open-loop drag reduction study of a turbulent boundary layer. The actuation mechanisms of 38 spanwise traveling transversal surface waves are investigated. MAO compares and classifies these actuated flows in agreement with physical intuition. For

$\ddagger$ Present address : Department of Mechanical Engineering, Massachusetts Institute of Technology, 77 Massachusetts Avenue, Cambridge, MA 02139, USA

$\dagger$ Email address for correspondence: rishabhishar.bemech14@pec.edu.in 
instance, the first feature coordinate of the attractor proximity map correlates with drag for the fluidic pinball and for the turbulent boundary layer. MAO has a large spectrum of potential applications ranging from a quantitative comparison between numerical simulations and experimental particle-image velocimetry data to the analysis of simulations representing a myriad of different operating conditions.

\section{Introduction}

In this study, we propose arguably the first general metric between attractor data from different operating conditions. With attractor data, we refer to an ensemble of statistically representative flow snapshots which allow for the computation of statistical moments and resolve coarse-grained coherent structures. Here, attractor is understood in a nonlinear dynamics sense for dissipative systems, i.e., a subset of the state space to which all solutions converge independently of the initial condition (see, e.g., Schuster 1988). The existence of a single global attractor is implicitly assumed in statistical fluid mechanics. Otherwise, statistical moments may have multiple values depending on the initial conditions. The focus on attractor data is not requested by the metric but simplifies the first demonstration of its usefulness.

In particular, we propose an unsupervised comparison methodology with little subjective bias. This methodology is exemplified for two configurations with associated many open-loop actuations each. The first example is the two-dimensional fluidic pinball, i.e., the flow around three stationary rotating circular cylinders (Noack et al. 2016). Unlike the single rotating cylinder, the fluidic pinball exhibits rich spatial-temporal dynamics under different actuation laws at similarly low computational cost (Noack \& Morzyński 2017). The second example demonstrates the applicability to three-dimensional wallbounded turbulent flow, namely the turbulent boundary layer actuated with a spanwise transversely traveling surface waves (Meysonnat et al. 2016).

Most fluid mechanics publications contain a comparison of flows from different sources, e.g. experiments versus simulations or at various operating conditions, e.g. optimal control versus the unforced benchmark.

In an engineering application, this comparison is easily performed for a single global solution parameter of interest, e.g. drag for a car, lift for an airfoil, mixing for a combustor or far-field noise of a jet engine.

These comparisons of performance parameters are simple but provide a limited assessment of the flow physics. For instance, Reynolds-averaged Navier-Stokes (RANS) simulations of cylinder wakes may predict well the drag coefficient at low Reynolds number. However, RANS computations predict the von Kármán vortex street to dissipate far too quickly in streamwise direction. A commonly used and more refined comparison includes the statistical moments of the flow field, or at least transverse or streamwise velocity profiles. The comparison of statistical moments is straightforward using a corresponding Hilbert space norm. This comparison is more detailed than employing a single order parameter. Yet, it excludes spatial-temporal dynamics of coherent structures.

Coherent structures are often visible to the naked eye, as beautifully depicted for wakes over 500 years ago by Leonardo da Vinci. Their quantification has been subject of thousands of publications and many disputes. Vortices provide important dynamical insight into geometrically simple two- and three-dimensional flows and have been the cornerstone of early reduced-order modeling efforts starting with the famous Helmholtz vortex laws in 1869 (see, e.g. Lugt 1996). Data-driven vortex identifications have been proposed by Jeong \& Hussain (1995) for snapshots and by Haller (2005) for flow histories. 
These frequently cited publications represent-pars pro toto-myriad of other feature analyses, e.g. Galilean invariant snapshot topology (Kasten et al. 2016).

An alternative approach is a reduced-order representation by expansions in terms of global modes. Proper orthogonal decomposition (Berkooz et al. 1993), dynamic mode decomposition (Rowley et al. 2009; Schmid 2010), stability eigenmodes (Theofilis 2011) or plain temporal Fourier expansions may serve as examples. These modes provide important insight into physical mechanisms of the coherent structure dynamics. Yet, one cannot expect the modes of different sources to coincide or even to be similar. In addition, the energy resolved by low-order turbulence representations is often much smaller than the energy of the unresolved stochastic fluctuations.

The analyses of local features and expansions in terms of global modes pose significant challenges for an automated comparison of different attractor data. In this study, we neither follow the Galerkin method nor the vortex modeling approach. Instead, the distance between two data sets is, roughly speaking, geometrically characterized by the average distance between each snapshot of one attractor to the closest snapshot of the other attractor. In characterizing the overlap and disjoint regions of both attractors by selected flow states, we follow the pioneering clustering approach of Burkardt et al. (2006) in fluid dynamics. Clustering implies that similar snapshots are put into 'bins' represented by a centroid. The centroid can be understood as a flow state averaged over all elements in the same 'bin'. Shared centroids span the overlap region of both attractors and disjoint centroids illustrate different attractor regions. The comparison methodology is augmented by powerful feature extraction from machine learning. The beauty and ingredients of this approach will be elucidated in the following section.

For the proposed framework, we choose a direct numerical simulation of the fluidic pinball, i.e., the flow around three equal circular cylinders with centers on an equilateral triangle pointing in upstream direction (Noack et al. 2016). The term 'fluidic pinball' is owed to the possibility of moving fluid particles like balls in a conventional pinball machine by suitably rotating the cylinders. The pinball configuration includes most wake stabilization strategies with suitable rotation of the three cylinders. Examples include phasor control (Roussopoulos 1993), aerodynamic boat tailing (Geropp 1995; Geropp \& Odenthal 2000; Barros et al. 2016), base bleed (Wood 1964; Bearman 1967), highfrequency forcing (Thiria et al. 2006; Oxlade et al. 2015) and low-frequency forcing (Pastoor et al. 2008). Another possibility is to deflect the wake via the Magnus effect. In this study, we compare the unforced reference and six open-loop actuation mechanisms.

Wakes or free-shear flows downstream of blunt bodies often possess dominant structures as opposed to wall-bounded shear flows whose distributions of the spatial and temporal scales exhibit smooth broadband spectra. As a challenging benchmark, the comparison methodology is also applied to data from a large-eddy simulation of a zero pressure-gradient boundary layer over a surface that undergoes a transversal spanwise traveling wave motion, i.e., the wall is deflected in the wall-normal direction. The canonical fully turbulent boundary layer flow is an interesting test case to study the impact of wall motion on friction drag. The results of this simple geometry are to a certain extent transferable to problems such as airfoil flows where the boundary layer varies in the streamwise direction. Besides the well-known passive control approaches, e.g., riblets (García-Mayoral \& Jiménez 2011), a wide range of active drag reduction methods has been investigated in the past three decades. To name a few, Jung et al. (1992) achieved high relative drag reduction using in-plane spanwise wall oscillations, $\mathrm{Du}$ et al. (2002) applied a traveling wave-like body force in the spanwise direction to lower the friction drag, and Zhao et al. (2004) extended the idea by an flexible wall 
approach. A good overview of active drag reduction approaches is given by Quadrio (2011).

Spanwise traveling transversal surface waves have been investigated experimentally (Itoh et al. 2006; Tamano \& Itoh 2012; Li et al. 2015) and numerically (Klumpp et al. 2010b; Koh et al. 2015b; Meysonnat et al. 2016). Drag reductions of the order of $10 \%$ were achieved. The physical mechanism of this active control is the generation of a secondary near-wall flow field in the wall-normal and spanwise direction through a, for example, sinusoidal up- and down motion of the wall to interrupt the near-wall cycle of the turbulent shear flow and as such to redistribute the turbulent scales. The main parameters for the sinusoidal wave actuation are wavelength, amplitude, and frequency. It goes without saying that due to the non-linear interaction between the wall-motion parameters and the wall-shear stress, i.e., the friction drag, it is quite a challenge to efficiently determine for a given flow, i.e., predefined freestream Reynolds number, the optimum parameter settings to minimize the wall-shear stress distribution. In this study, the large-eddy simulation data for a non-actuated turbulent boundary layer flow constitutes the reference problem. The drag reduction is studied for 37 transverse surface wave actuations which vary in wavelength, amplitude, and frequency.

The structure of the manuscript is as follows. In $\S 2$, the employed fluidic pinball simulation and the actuated turbulent boundary layer flow are described. The corresponding data includes converged data of the 7 attractors and of the 38 parameter variations of the traveling wave. In $\S 3$, the comparison methodology for attractor data is outlined. The proposed approach is exemplified for all operating conditions of the fluidic pinball in $\S 4$ and for all actuations of the turbulent boundary layer in $\S 2.2$. Section $\S 6$ summarizes this study and outlines future directions of research.

\section{Plant configurations}

The comparison methodology MAO is applied to two configurations with many data sets from open-loop actuation studies. A two-dimensional fluidic pinball configuration $(\S 2.1)$ is computationally inexpensive and allows an extensive visualization of the flow quantities while exhibiting a complex behavior. The second set from an open-loop drag reduction study of a turbulent boundary layer (§ 2.2) shall allow to assess MAO for complex three-dimensional flow data with a large range of scales and frequencies.

\subsection{Fluidic pinball simulation}

In this section, the computation of the employed flow data is described. In $\S 2.1 .1$, the configuration of the fluidic pinball is introduced. The corresponding direct NavierStokes solver is described in $\S 2.1 .2$. Finally, the fluidic pinball simulation of seven openloop actuations is detailed. These flow data are subjected to the proposed comparison methodology of the next section.

\subsubsection{Fluidic pinball configuration}

In the following, the considered two-dimensional flow control configuration is described. Three equal circular cylinders with radius $R$ are placed parallel to each other in a viscous incompressible uniform flow at speed $U_{\infty}$ (see Fig. 1). The centers of the cylinders form an equilateral triangle with side length $3 R$, symmetrically positioned with respect to the flow. The leftmost triangle vertex points upstream, while the rightmost side is orthogonal to the oncoming flow. Thus, the transverse extent of the three-cylinder configuration is given by $5 R$.

This flow is described in a Cartesian coordinate system where the $x$-axis points in 


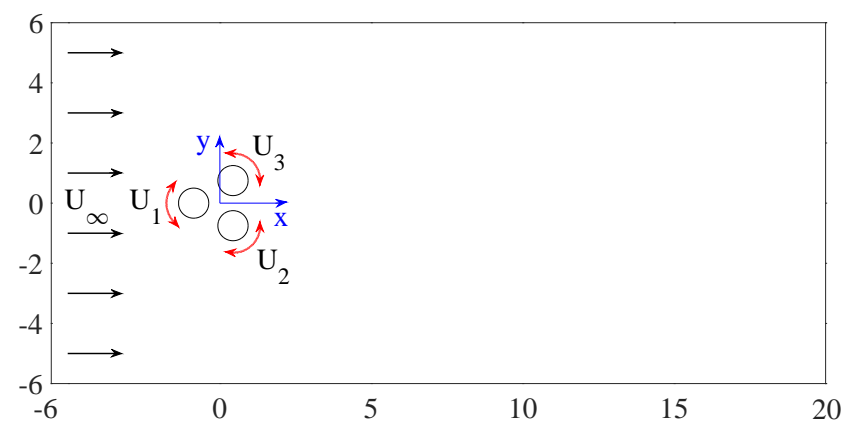

Figure 1. Configuration of the fluidic pinball. The Cartesian coordinate system $(x, y)$ is depicted at the center of the cylinders.

the direction of the flow, the $z$-axis is aligned with the cylinder axes, and the $y$-axis is orthogonal to both (see figure 1). The origin $\mathbf{0}$ of this coordinate system coincides with the geometric center of the cylinder triangle. The location is denoted by $\boldsymbol{x}=(x, y, z)=$ $x \boldsymbol{e}_{x}+y \boldsymbol{e}_{y}+z \boldsymbol{e}_{z}$, where $\boldsymbol{e}_{x, y, z}$ are unit vectors pointing in the direction of the corresponding axes. Analogously, the velocity reads $\boldsymbol{u}=(u, v, w)=u \boldsymbol{e}_{x}+v \boldsymbol{e}_{y}+w \boldsymbol{e}_{z}$. The pressure is denoted by $p$ and the time by $t$. In the following, we assume a two-dimensional flow, i.e., no dependence of any flow quantity on $z$ and vanishing spanwise velocity $w \equiv 0$.

The Newtonian fluid is characterized by a constant density $\rho$ and kinematic viscosity $\nu$. In the following, all quantities are assumed to be non-dimensionalized with cylinder diameter $D=2 R$, the velocity $U_{\infty}$ and the fluid density $\rho$. The corresponding Reynolds number is $R e_{D}=100$ where $R e_{D}=U_{\infty} D / \nu$. The Reynolds number based on the transverse length $L=5 D$ is 2.5 times larger. The non-dimensionalization with respect to the diameter is more common for clusters of cylinders (Hu \& Zhou 2008a,b; Bansal \& Yarusevych 2017) and will be adopted in the following. With this non-dimensionalization, the cylinder axes are located at

$$
\begin{array}{ll}
x_{F}=-\sqrt{3} / 2, & y_{F}=0, \\
x_{B}=\sqrt{3} / 4, & y_{B}=-3 / 4, \\
x_{T}=\sqrt{3} / 4, & y_{T}=+3 / 4 .
\end{array}
$$

Here, and in the following, the subscripts ' $F$ ', ' $B$ ' and ' $T$ ' refer to the front, bottom and top cylinder. An alternate reference is the subscripts 1, 2, 3 for the front, bottom, and top cylinder, respectively. The numbering is in mathematically positive orientation.

The incompressibility condition reads

$$
\nabla \cdot \boldsymbol{u}=0
$$

where $\nabla$ represents the Nabla operator. The evolution is described by the Navier-Stokes equations,

$$
\partial_{t} \boldsymbol{u}+\nabla \cdot \boldsymbol{u} \otimes \boldsymbol{u}=-\nabla p+\frac{1}{R e_{D}} \triangle \boldsymbol{u},
$$

where $\partial_{t}$ and $\triangle$ denote the partial derivative with respect to time $t$ and the Laplace operator, respectively. The dot '.' and dyadic product sign ' $\otimes$ ' refer to inner and outer tensor products.

Without forcing, the boundary conditions comprise a no-slip condition on the cylinder and a free-stream condition in the far field:

$$
\boldsymbol{u}=\mathbf{0} \text { on the cylinder and } \boldsymbol{u}=\boldsymbol{e}_{x} \text { at infinity. }
$$




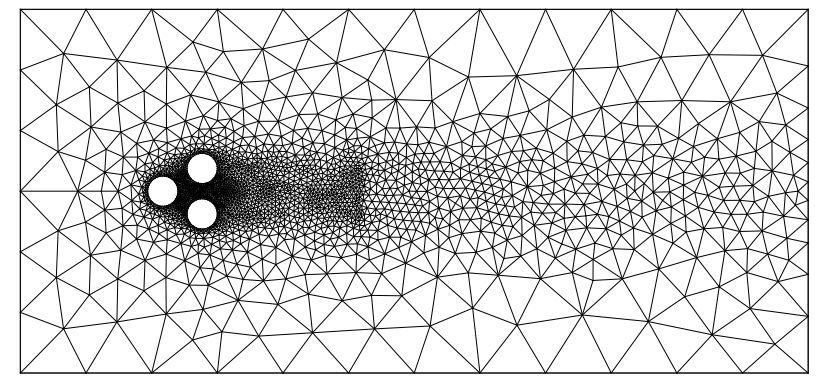

FIGURE 2. Grid of the fluidic pinball simulation.

As initial condition, we chose the unstable steady Navier-Stokes solution $\boldsymbol{u}_{s}(\boldsymbol{x})$. This solution is computed with a Newton search algorithm, like in (Noack et al. 2003). Vortex shedding is kick-started with cylinder rotations in the first period.

The forcing is exerted by rotation of the cylinders with circumferential velocities $b_{1}=$ $U_{1}=U_{F}, b_{2}=U_{2}=U_{B}$ and $b_{3}=U_{3}=U_{T}$ for the front, bottom and top cylinder, respectively. The actuation command $\boldsymbol{b}=\left(b_{1}, b_{2}, b_{3}\right)=\left(U_{1}, U_{2}, U_{3}\right)$ is preferably used for control theory purposes (Brunton \& Noack 2015; Duriez et al. 2016) while $\left(U_{F}, U_{B}, U_{T}\right)$ are more natural for a discussion of physical mechanisms. The actuation is conveniently expressed with the vector cross product ' $x$ ':

$$
\boldsymbol{u}=2 U_{i} \boldsymbol{x} \times \boldsymbol{e}_{z} \text { on the } i \text { th cylinder. }
$$

The factor 2 counterbalances the non-dimensional radius $1 / 2$.

We like to refer to this configuration as fluidic pinball as the rotation speeds allow one to change the paths of the incoming fluid particles like flippers manipulate the ball of a conventional pinball machine. The front cylinder rotation may determine if the fluid particle passes by on the upper or lower side of the cylinder, while the top and bottom cylinder may guide the particle through the interior.

\subsubsection{Direct Navier-Stokes solver}

The chosen computational domain is bounded by the rectangle $[-6,20] \times[-6,6]$ and excludes the interior of the cylinders:

$$
\Omega=\left\{(x, y):-6 \leqslant x \leqslant 20 \wedge|y| \leqslant 6 \wedge\left(x-x_{i}\right)^{2}+\left(y-y_{i}\right)^{2} \geqslant 1 / 4, i=1,2,3\right\} .
$$

This flow domain is discretized on an unstructured grid with 4225 triangles and 8633 vertices (see Fig. 2). This discretization optimizes the speed of the numerical simulation while keeping the accuracy at an acceptable level. Increasing the number of triangles by a factor 4 yields virtually indistinguishable results. The Navier-Stokes equation is numerically integrated with an implicit Finite-Element Method (Noack et al. 2003, 2016). The numerical integration is second-order accurate in space and third-order accurate in time. This direct numerical simulation has a companion experiment at turbulent Reynolds numbers $R e_{D} \approx 4000-6000$ (Raibaudo et al. 2017).

\subsubsection{Attractor data}

We simulate seven actuations in a single simulation starting at $t=0$ with the unstable steady Navier-Stokes solution. Each phase is associated with one control law and lasts 100 convective time units. Figure 3 provides a preview of the simulation which is detailed in the following.

In the first phase, vortex shedding is kick-started with a motion of the front cylinder, 


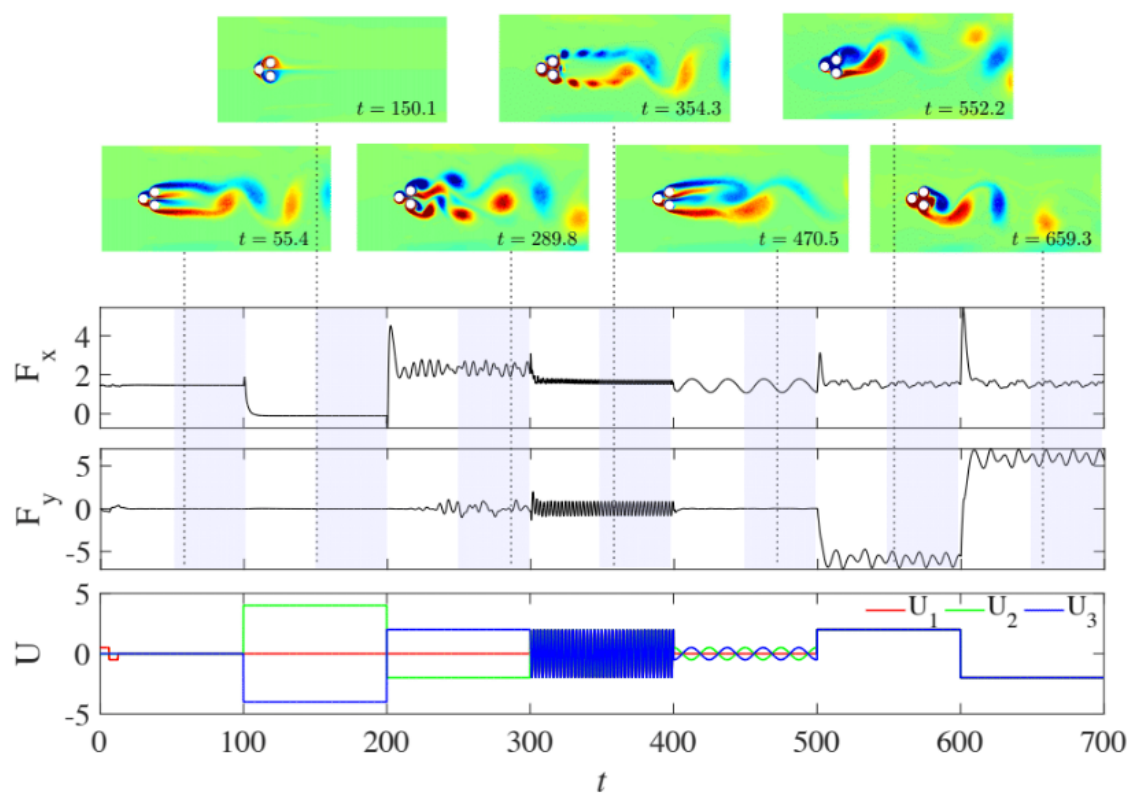

FiguRE 3. Operating conditions of the fluidic pinball simulation. Bottom: time series of the actuation commands for each cylinder over all phases together. Top: the flow of each phase is illustrated with a vorticity snapshot corresponding to maximum lift or minimum drag of the converged phase. Center: the time series of the total drag $F_{x}$ and total lift $F_{y}$ are displayed.

$$
\begin{aligned}
& U_{F}=\left\{\begin{array}{lr}
1 / 2 & t \leqslant 6.25 \\
-1 / 2 & 6.25<t \leqslant 12.5 \\
0 & \text { otherwise }
\end{array}\right. \\
& U_{B}=-U_{T}=0 .
\end{aligned}
$$

The imposed period 12.5 corresponds to a Strouhal number of 0.2 based on the transverse width $5 R$. Without this kick-start, the onset of vortex shedding may require hundreds of shedding periods depending on accuracy of the steady solution and the truncation errors in the Navier-Stokes solver. In the second phase, strong boat tailing with symmetric cylinder rotation of the upper and lower cylinder pushes the separation close to the $x$-axis and completely suppresses vortex shedding,

$$
U_{F}=0, \quad U_{B}=-U_{T}=4 .
$$

In the third phase, base-bleed is enforced with opposite cylinder motion:

$$
U_{F}=0, \quad U_{B}=-U_{T}=-2 .
$$

This actuation widens the wake and pushes vortex formation downstream. In the fourth phase, symmetric high-amplitude, high-frequency actuation energizes both shear layers:

$$
U_{F}=U_{B}=U_{T}=2 \cos (10 \pi t / 12.5) .
$$

The frequency corresponds roughly to five times the one of natural vortex shedding, following Thiria et al. (2006) for a single cylinder. In the fifth phase, symmetric lowamplitude, low-frequency forcing delays vortex shedding:

$$
U_{F}=0, \quad U_{B}=-U_{T}=\frac{1}{2} \cos (\pi t / 12.5) .
$$




\begin{tabular}{rlll}
\hline Phase & Time & Actuation mechanism & Control law \\
\hline I & $t \leqslant 100$ & unforced reference & $U_{F}=U_{B}=U_{T}=0$ for $t>12.5$, see $(2.6)$ \\
II & $t \in(100,200]$ & boat tailing & $U_{F}=0, U_{B}=-U_{T}=4$ \\
III & $t \in(200,300]$ & base bleed & $U_{F}=0, U_{B}=-U_{T}=-2$ \\
IV & $t \in(300,400]$ & high-frequency forcing & $U_{F}=U_{B}=U_{T}=2 \cos (10 \pi t / 12.5)$ \\
V & $t \in(400,500]$ & low-frequency forcing & $U_{F}=0, U_{B}=-U_{T}=1 / 2 \cos (\pi t / 12.5)$ \\
VI & $t \in(500,600]$ & positive Magnus effect & $U_{F}=U_{B}=U_{T}=2$ \\
VII & $t \in(600,700]$ & negative Magnus effect & $U_{F}=U_{B}=U_{T}=-2$ \\
\hline
\end{tabular}

TABLE 1. Summary of the different control phases of the fluidic pinball simulation and the associated actuation mechanism.

Low amplitudes were found to be most effective in stabilizing the wake in a parametric study Rolland (2017). In the sixth phase, a uniform rotation of all cylinders deflects the wake upwards via the Magnus effect,

$$
U_{F}=U_{B}=U_{T}=2 .
$$

In the seventh and last phase, the opposite Magnus effect is imposed,

$$
U_{F}=U_{B}=U_{T}=-2 .
$$

Table 1 summarizes all control laws for later reference $\dagger$.

The attractor data contains time-resolved snapshots from the last 50 convective units of each phase. These snapshots are equidistantly sampled with a time step of 0.1 , i.e., each phase is represented by 500 velocity fields. The first 50 convective time units correspond to 2.5 downwash times - enough for the transient dynamics to die out. We decided to perform one simulation with different control laws since the transients reveal the robustness of the posttransient phase.

\subsection{Actuated turbulent boundary layer}

The generation of data of the turbulent boundary layer flow over a surface undergoing a transversal spanwise traveling wave motion is described in this section. First, the configuration of the boundary layer flow is described in $\S 2.2 .1$. Then, the numerical method of the flow solver is presented in $\S 2.2 .2$. Finally, the collection of attractor data from 38 simulations with varying actuation parameters is detailed in $\S 2.2 .3$.

\subsubsection{Boundary layer configuration}

The zero-pressure gradient (ZPG) turbulent boundary layer flow actuated by a sinusoidal wall motion is defined in a Cartesian domain with the $x$-axis corresponding to the mean flow direction, the $y$-axis pointing into wall-normal direction, and the $z$-axis in the spanwise direction. Positions are denoted by $\mathbf{x}=(x, y, z)$ and the corresponding velocities by $\mathbf{u}=(u, v, w)$, the pressure is given by $p$ and the density by $\rho$. The governing equations of the flow are the unsteady compressible Navier-Stokes equations and the thermal and caloric state equations. The heat flux is described by Fourier's law and the temperate dependence of the fluid viscosity is given by Sutherland's law. Unlike standard ZPG turbulent boundary layer flow, the actuated flow is statistically three-dimensional due to the wave propagating in the $z$-direction. The flow variables are non-dimensionalized using the flow quantities at rest, the speed of sound $a_{0}$, and the momentum thickness

$\dagger$ A visualization of the whole simulation can be found on http://fluidicpinball.com. 
of the boundary layer at $x_{0}=0$, such that $\theta\left(x_{0}=0\right)=1$. The momentum thickness based Reynolds number is $R e_{\theta}=1,000$ at $x_{0}$ where $R e_{\theta}=u_{\infty} \theta / \nu$. The Mach number is $M=0.1$, i.e., the flow is nearly incompressible.

An overview of the setup is given in figure 4 . The dimensions of the physical domain are $L_{x}=190 \theta, L_{y}=105 \theta$, and $L_{z}=21.65 \theta$. At the inflow of the domain, the reformulated synthetic turbulence generation (RSTG) method by Roidl et al. (2013) is used to prescribe a fully turbulent inflow distribution with an adaptation length of less than five boundary-layer thicknesses $\delta_{99}$, such that a natural turbulence state is achieved at $x_{0}$, which marks the onset of the actuation. Characteristic outflow conditions are applied at the downstream and upper boundary of the domain and periodic conditions are used in the spanwise direction. On the wall, no-slip conditions are imposed and the wall motion is described by

$$
\left.y^{+}\right|_{\text {wall }}\left(z^{+}, t^{+}\right)=g(x) A^{+} \cos \left(\frac{2 \pi}{\lambda^{+}} z^{+}+\frac{2 \pi}{T^{+}} t^{+}\right),
$$

where $A^{+}=A u_{\tau} / \nu$ is the amplitude, $\lambda^{+}=\lambda u_{\tau} / \nu$ the wavelength, and $T^{+}=T u_{\tau}^{2} / \nu$ the period of the traveling wave in inner coordinates, i.e., scaled by the kinematic viscosity $\nu$ and the friction velocity $u_{\tau}\left(x_{0}\right)$ of the non-actuated reference case. The piecewise defined function

$$
g(x)= \begin{cases}0 & \text { if } \quad x<-5 \\ \frac{1}{2}\left[1-\cos \left(\frac{\pi(x+5)}{10}\right)\right] & \text { if }-5 \leqslant x<5 \\ 1 & \text { if } 5 \leqslant x<130 \\ \frac{1}{2}\left[1+\cos \left(\frac{\pi(x-130)}{10}\right)\right] & \text { if } 130 \leqslant x<140 \\ 0 & \text { otherwise }\end{cases}
$$

enables a smooth streamwise transition from a flat non-actuated to an actuated wall and vice versa. Apart from the reference case without any wall actuation, i.e., $A^{+}=0$ and $T^{+}=0,37$ parameter combinations of $\lambda^{+}, T^{+}$, and $A^{+}$are considered (see table 3 ). Most parameter points were generated using latin hypercube sampling.

The physical domain is discretized by a structured body-fitted grid with a resolution of $\Delta x^{+}=12$ in the $x$-direction, $\left.\Delta y^{+}\right|_{\text {wall }}=1.0$ in the $y$-direction using gradual coarsening with increasing distance from the wall, and $\Delta z^{+}=4.0$ in the $z$-direction. This nearly DNS-like resolution guarantees to capture all relevant turbulent scales and allows a smooth representation of the wavy wall. In total, the grid consists of $n=732 \times 131 \times 250 \approx 24 \cdot 10^{6}$ cells. The details of the flow conditions and grid parameters are summarized in table 2 .

\subsubsection{Large-eddy simulation solver}

The actuated turbulent boundary layer flow is simulated using a finite volume approximation of the unsteady compressible Navier-Stokes equation on a structured body-fitted mesh. A second-order accurate formulation of the inviscid fluxes using the advection upstream splitting method (AUSM) by Liou \& Steffen (1993) is applied. The cell-surface values of the flow quantities are reconstructed from the surrounding cell-center values using a Monotone Upstream Scheme for Conservation Laws (MUSCL) type strategy. The viscous fluxes are discretized by a modified cell-vertex scheme at second-order accuracy. The time integration is performed by a second-order accurate five-stage Runge-Kutta scheme, rendering the overall discretization second-order accurate. The subgrid scales in the LES are implicitly modeled following the monotonically integrated large-eddy 


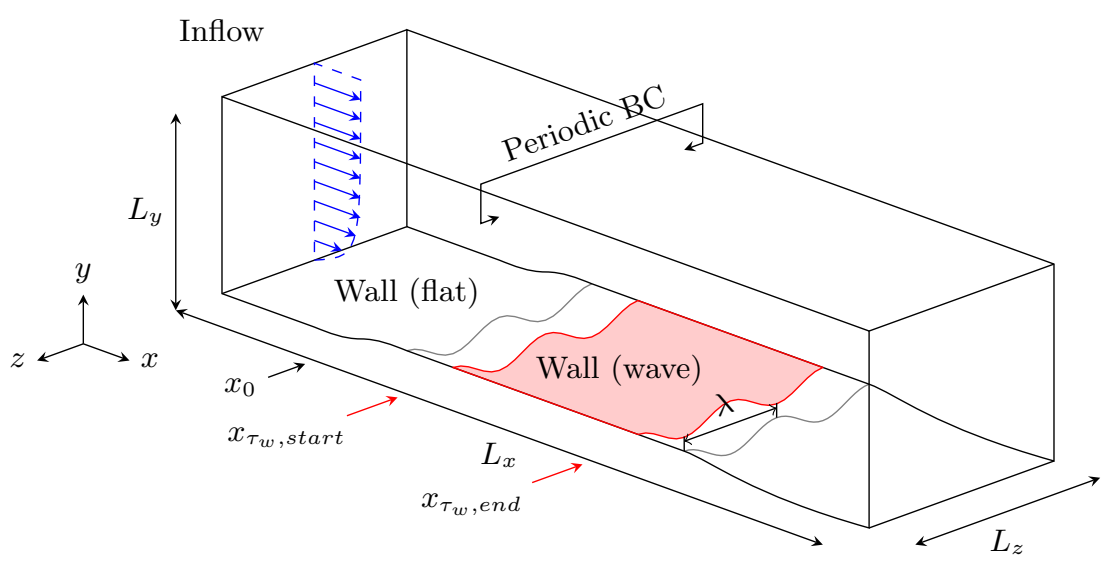

FiguRE 4. Overview of the physical domain of the actuated turbulent boundary layer flow, where $L_{x}, L_{y}$, and $L_{z}$ are the dimensions of the domain in the Cartesian directions, $\lambda$ is the wavelength of the spanwise traveling wave, $x_{0}$ marks the onset of the actuation, and $x_{\tau_{w}, \text { start }}$ and $x_{\tau_{w} \text {,end }}$ denote the interval of the integration of the wall-shear stress $\tau_{w}$.

\begin{tabular}{ll} 
Parameter & Value \\
\hline$M_{\infty}$ & 0.1 \\
$\operatorname{Re}_{\theta}$ & 1,000 \\
$\Delta x^{+}$ & 12.0 \\
$\Delta y_{\text {wall }}^{+}$ & 1.0 \\
$\Delta z^{+}$ & 4.0 \\
$\left.\delta_{99}(x=0.0)\right)$ & 8.638 \\
$\delta_{1}(x=0.0)$ & 1.488 \\
$\theta(x=0.0)$ & 1.0 \\
$L_{x} \times L_{y} \times L_{z}$ & $190.15 \times 104.91 \times 21.65$ \\
$n_{\text {cells }}$ & $732 \times 131 \times 250 \approx 24 \cdot 10^{6}$ \\
$x_{\tau_{w}, \text { start }}$ & 50.0 \\
$x_{\tau_{w}, \text { end }}$ & 100.0 \\
\hline
\end{tabular}

TABLE 2. Flow and grid parameters of the non-actuated reference case and the 37 actuated cases.

simulation approach (Boris et al. 1992), i.e., the numerical dissipation of the AUSM scheme models for the viscous dissipation of the high wavenumber turbulence spectrum (Meinke et al. 2002). Thus, the small-scale structures are not explicitly resolved and the grid is used as a spatial filter resolving the large energy-containing structures in the inertial subrange. To capture the temporal variation of the geometry, the NavierStokes equations are written in the Arbitrary Lagrangian-Eulerian (ALE) formulation (Hirt et al. 1997) such that the actuated wall can be represented by an appropriate mesh deformation. Additional volume fluxes are determined to satisfy the Geometry Conservation Law (GCL).

The numerical method has been thoroughly validated by computing a wide variety of internal and external flow problems (Rütten et al. 2005; Alkishriwi et al. 2006; Renze et al. 2008; Statnikov et al. 2017). Analyses of drag reduction in turbulent boundary layer flow have been performed for riblet structured surfaces (Klumpp et al. 2010a) and for traveling transversal surface waves (Klumpp et al. 2011; Koh et al. 2015b,a; Meysonnat 


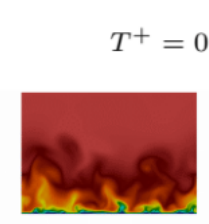

$u$

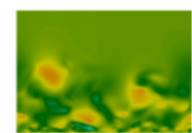

$w$

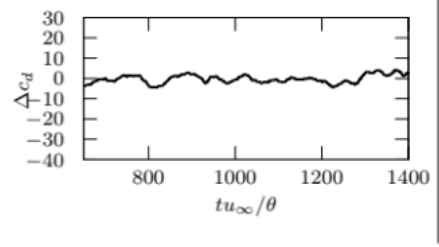

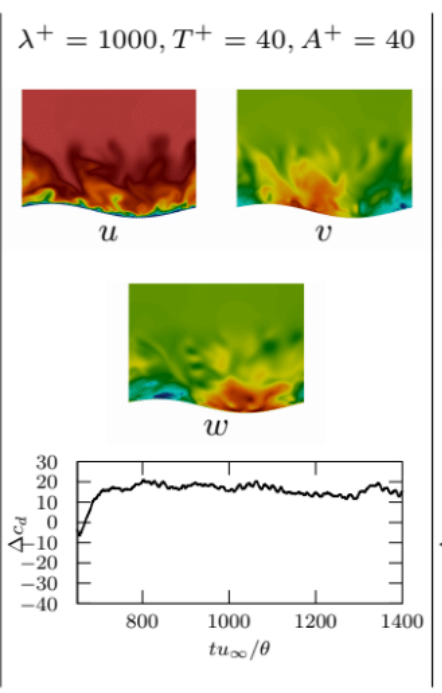

$\lambda^{+}=200, T^{+}=20, A^{+}=30$

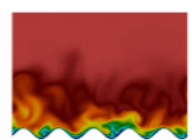

$u$
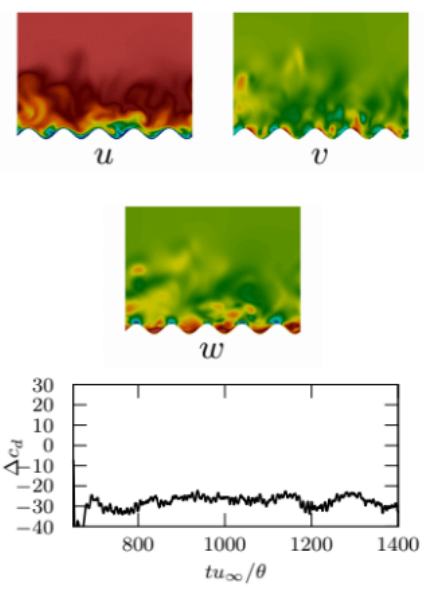

FIGURE 5. Illustration of the turbulent boundary layer flow: (left) non-actuated reference case $N_{1}$, (center) actuated case with highest drag reduction $N_{24}$, and (right) actuated case with lowest drag reduction $N_{2}$; (top) contour plots of the instantaneous Cartesian velocity components $u, v$, and $w$ in a $y-z$ plane at $x / \theta \approx 65$; (bottom) time evolution of the instantaneous relative drag reduction $\Delta c_{d}$.

et al. 2016). The quality of the results confirms the validity of the approach for the current flow problem.

\subsubsection{Attractor data}

First, the simulation of the reference setup is run for $t u_{\infty} / \theta \approx 650$ convective units until a quasi-steady state of the drag evolution is observed. Then, the average drag of the reference setup is measured for the next $\Delta t u_{\infty} / \theta \approx 1000$ convective units. Subsequently, the actuated cases are initiated using an intermediate solution from the reference case and a quick transition from the flat wall to the fully deflected wall is enforced. When a new quasi-steady state of the friction drag is obtained, i.e., after $\Delta t u_{\infty} / \theta \approx 150$ convective units, the drag of each actuated case is averaged over a period of $\Delta t u_{\infty} / \theta \approx 800$ convective units. The relative drag reduction for the i-th test case

$$
\Delta c_{d, \mathrm{i}}=\frac{\int_{A, \text { na }} \tau_{w, \text { na }} d A-\int_{A, \mathrm{i}} \tau_{w, \mathrm{i}} d A}{\int_{A, \text { na }} \tau_{w, \text { na }} d A} \cdot 100
$$

is computed by integrating the wall-shear stress distribution $\tau_{w}$ over the wetted surface $A$ in the interval $x \in\left[x_{\tau_{w}, \text { start }}, x_{\tau_{w}, \text { end }}\right]$ of the non-actuated reference (subscript $n a$ ) and the actuated setups (subscript $i$ ). The values of the relative drag reduction $\Delta c_{d}$ and the skin-friction reduction $\Delta c_{f}$, i.e., the drag reduction without considering the increase in the wetted surface, of the various amplitude, wavenumber, and period prescriptions are listed in table 3. An exemplary illustration of the impact of the moving surface on the near-wall velocity field and the friction drag development is given in figure 5 for the reference case $N_{1}$, the case with the highest drag reduction $N_{24}$, and the case with the lowest drag reduction $N_{2}$. In figure 6 , the variation of the turbulent structures in the near-wall region of these three cases is shown. The comparative juxtaposition of the data evidences the decrease of the turbulent structures for the $N_{24}$ case. 


\begin{tabular}{|c|c|c|c|c|c|}
\hline$N$ & $\lambda^{+}$ & $T^{+}$ & $A^{+}$ & $\Delta c_{d}$ & $\Delta c_{f}$ \\
\hline 1 & - & - & - & - & - \\
\hline 2 & 200 & 20 & 30 & -27.57 & -6.82 \\
\hline 3 & 200 & 30 & 21 & -1.13 & 8.16 \\
\hline 4 & 200 & 40 & 30 & -9.43 & 8.37 \\
\hline 5 & 200 & 50 & 45 & -26.58 & 8.93 \\
\hline 6 & 200 & 60 & 30 & -9.89 & 7.98 \\
\hline 7 & 200 & 70 & 14 & -0.79 & 3.70 \\
\hline 8 & 200 & 70 & 38 & -17.42 & 9.23 \\
\hline 9 & 200 & 100 & 28 & -9.79 & 6.29 \\
\hline 10 & 500 & 20 & 30 & -0.17 & 3.18 \\
\hline 11 & 500 & 30 & 22 & 8.18 & 9.88 \\
\hline 12 & 500 & 40 & 21 & 6.41 & 7.99 \\
\hline 13 & 500 & 40 & 30 & 7.51 & 10.61 \\
\hline 14 & 500 & 60 & 30 & 3.77 & 7.00 \\
\hline 15 & 500 & 70 & 36 & 2.66 & 7.24 \\
\hline 16 & 500 & 70 & 64 & -10.52 & 3.56 \\
\hline 17 & 500 & 100 & 48 & -4.53 & 3.70 \\
\hline 18 & 1000 & 20 & 10 & 3.58 & 3.67 \\
\hline 19 & 1000 & 20 & 30 & 11.95 & 12.72 \\
\hline 20 & 1000 & 20 & 50 & -0.45 & 1.93 \\
\hline 21 & 1000 & 40 & 10 & 3.15 & 3.24 \\
\hline 22 & 1000 & 40 & 20 & 6.48 & 6.84 \\
\hline 23 & 1000 & 40 & 30 & 11.79 & 12.56 \\
\hline 24 & 1000 & 40 & 40 & 15.69 & 16.98 \\
\hline 25 & 1000 & 40 & 50 & 14.77 & 16.79 \\
\hline 26 & 1000 & 40 & 60 & 12.49 & 15.42 \\
\hline 27 & 1000 & 80 & 10 & 0.65 & 0.75 \\
\hline 28 & 1000 & 80 & 20 & 3.49 & 3.87 \\
\hline 29 & 1000 & 80 & 30 & 5.60 & 6.42 \\
\hline 30 & 1000 & 80 & 40 & 9.18 & 10.58 \\
\hline 31 & 1000 & 80 & 50 & 8.86 & 11.01 \\
\hline 32 & 1000 & 80 & 60 & 8.34 & 11.41 \\
\hline 33 & 1000 & 120 & 10 & 0.73 & 0.83 \\
\hline 34 & 1000 & 120 & 20 & -0.51 & -0.11 \\
\hline 35 & 1000 & 120 & 30 & 2.07 & 2.93 \\
\hline 36 & 1000 & 120 & 40 & 4.34 & 5.81 \\
\hline 37 & 1000 & 120 & 50 & 3.02 & 5.31 \\
\hline 38 & 1000 & 120 & 60 & 2.03 & 5.31 \\
\hline
\end{tabular}

TABLE 3. Actuation parameters of the turbulent boundary layer simulations, where each setup is denoted by a case number $N$. The quantity $\lambda^{+}$is the spanwise wavelength of the traveling wave, $T^{+}$is the period, and $A^{+}$is the amplitude, all given in inner units, i.e., non-dimensionalized with the kinematic viscosity $\nu$ and the friction velocity $u_{\tau}$. Each block includes setups with varying period and amplitude for a constant wavelength. The list includes the values of the averaged relative drag reduction $\Delta c_{d}$, and the averaged relative skin friction reduction $\Delta c_{f}$.

The snapshots for the computation of the POD modes are obtained in a subdomain spanning from $x=15 \theta$ to $x=120 \theta$, from the wall to a thickness of $y=15.4 \theta$, and over the full spanwise extent of the computational domain. That is, the subdomain covers the complete boundary layer over the actuated part of the wall, excluding the zones of spatial 


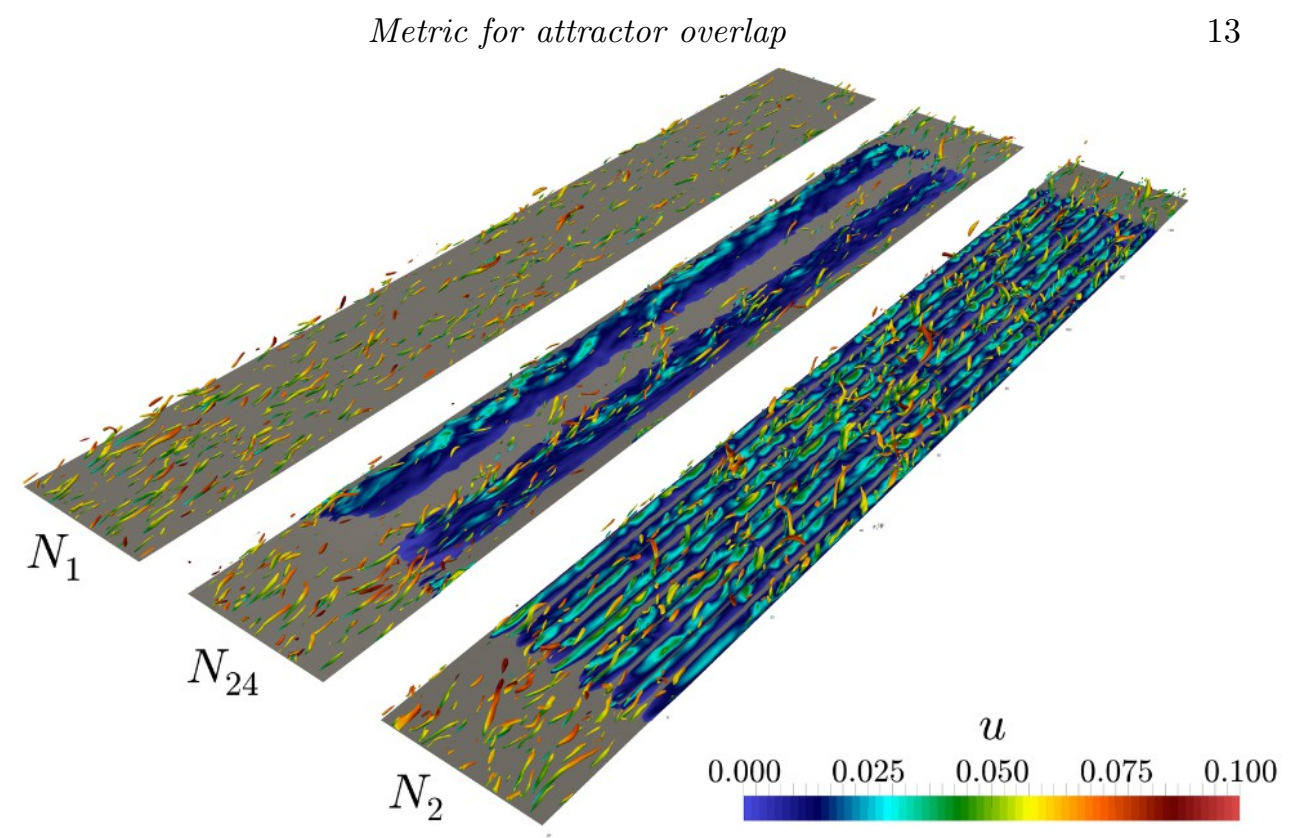

Figure 6. Contour plot of the $\lambda_{2}$-criterion (Jeong \& Hussain 1995), coloured by the instantaneous streamwise velocity, for three turbulent boundary layer flows; non-actuated reference case $N_{1}$, actuated highest drag reduction case $N_{24}$, actuated lowest drag reduction case $N_{2}$.

transition. The data are collected at the quasi-steady states with a sampling period of $\Delta t u_{\infty} / \theta \approx 0.94$, i.e., every $n=300$ iteration steps.

\section{Comparison methodology for different attractors}

In this section, we propose a comparison methodology for attractor data. The first constitutive element is a standard metric for snapshots as described in $\S 3.1$. In $\S 3.2$, this metric is generalized to attractor data and referred to as Metric for Attractor Overlap (MAO). In $\S 3.3$, the closeness of all attractors are featured in proximity maps.

A coarse-grained version of MAO is enabled by clustering (§ 3.4). This coarse-graining (§ 3.5) reduces the computational expense of the metric and gives visual access to select coherent structures which the attractors have in common, i.e., they overlap, or do not share, i.e., they are disjoint.

Figure 7 previews the proposed methodology. The top row illustrates the processing from the raw data of multiple attractors (left) to the proximity map (right) employing the snapshot MAO. The center row represents the coarse-grained version with clustering. Clustering opens the opportunity to pinpoint the overlap region of attractors to a few select velocity fields, i.e., shared centroids, as indicated in the bottom row.

\subsection{Distance between snapshots}

Let $\boldsymbol{u}(\boldsymbol{x})$ and $\boldsymbol{v}(\boldsymbol{x})$ be two velocity fields in the domain $\Omega$. We define the distance between these fields as

$$
D(\boldsymbol{u}, \boldsymbol{v})=\sqrt{\int_{\Omega} d \boldsymbol{x}\|\boldsymbol{u}(\boldsymbol{x})-\boldsymbol{v}(\boldsymbol{x})\|^{2}} .
$$




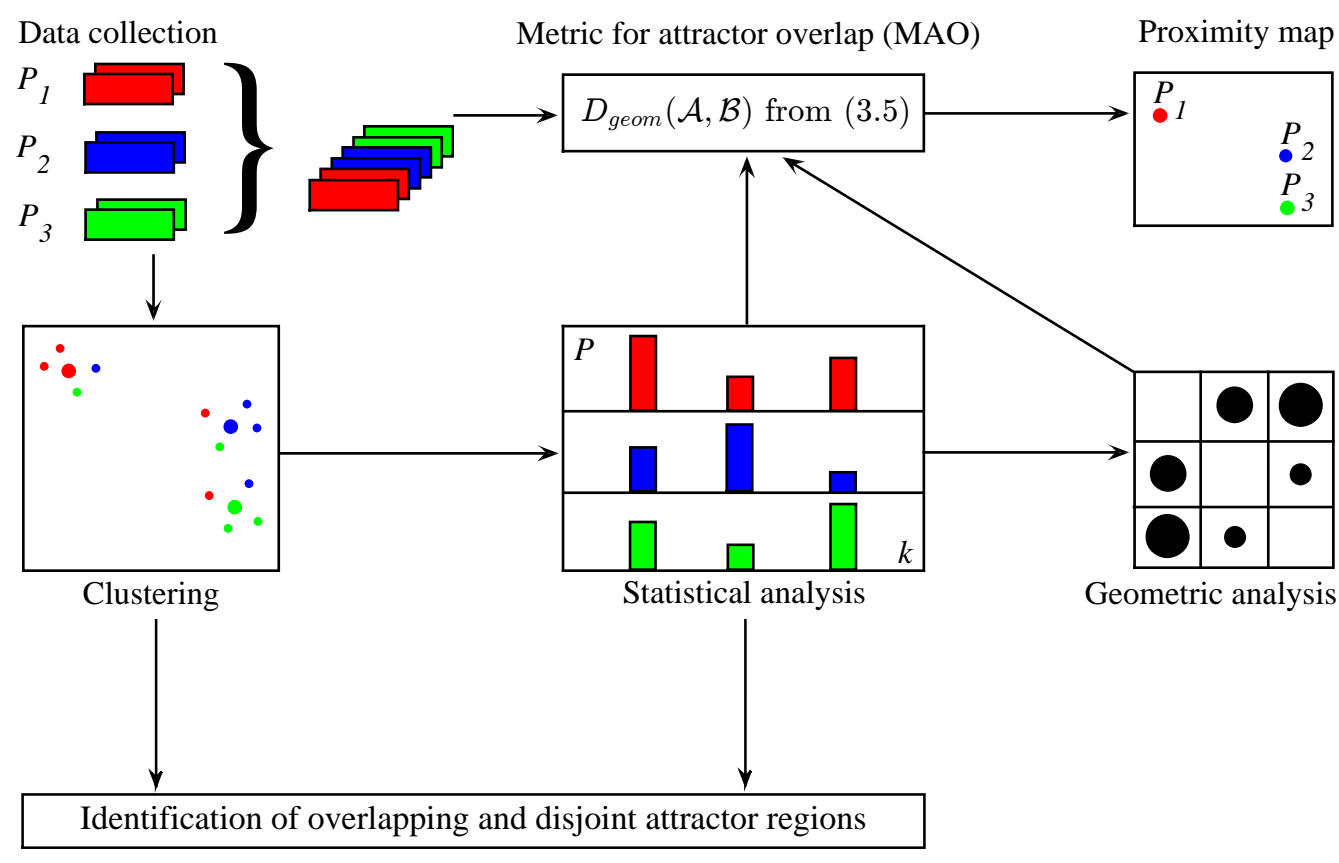

FiguRE 7. Schematic of the pipeline for the metric of attractor overlap and associated proximity maps. Here, $P_{1}, P_{2}$ and $P_{3}$ refer to data sets outlined in $\S 2$, i.e., sets of snapshots which are statistically representative for an attractor under a given operating condition. Of course, the number of data sets is typically much larger. $D$ is the metric of attractor overlap ( $\S 3.2)$. Its two arguments $P, Q$ represent data sets, like $P_{1}, P_{2}$ and $P_{3}$. The proximity map (top right) is explained in $\S 3.3$. The coarse-graining by clusters (center row) is discussed in $\S 3.4$ and the identification of overlapping and disjoint attractor regions (bottom row) is outlined in $\S 3.5$.

Here, $\|\cdot\|$ denotes the Euclidean norm. Note that this distance is based on the norm associated with the Hilbert space $\mathcal{L}^{2}(\Omega)$ of square-integrable functions. It fulfills all properties of a metric, like positive definiteness, commutativity, scaling with a real factor and triangle inequality. For the fluidic pinball data of $\S 2.1$, the distance measure uses the whole computational domain $\Omega$. For the turbulent boundary layer of $\S 2.2$, a subdomain over the whole actuated surface is chosen. The surface actuation leads to a small domain deformation. This deformation is neglected and the operations are performed in a stationary domain in which the grid points assume their the unactuated equilibrium location.

\subsection{Metric of attractor overlap}

In this section, we define a measure for the attractor similarity based on the snapshot configuration and the Hilbert-space metric. Loosely speaking, the difference between two attractors $\mathcal{A}$ and $\mathcal{B}$ - represented by their snapshot ensembles — is geometrically defined as the sum of the average distances between the snapshots of $\mathcal{A}$ to $\mathcal{B}$ and vice versa.

In the following, this quantity is defined. For simplicity, let us consider two attractors $\mathcal{A}$ and $\mathcal{B}$. Let $\mathcal{M}:=\left\{\boldsymbol{u}^{m}\right\}_{m=1}^{M}$ be the union of all snapshots from both attractors. For simplicity, we assume the generic case that all snapshots are pairwise different. The subset 
of $\mathcal{M}$ belonging to attractor $\mathcal{A}$ is defined by the characteristic function

$$
\chi_{\mathcal{A}}^{m}:= \begin{cases}1, & \text { if } \boldsymbol{u}^{m} \in \mathcal{A}, \\ 0, & \text { otherwise }\end{cases}
$$

The number of snapshots in $\mathcal{A}$ is given by $M_{A}:=\sum_{m=1}^{M} \chi_{\mathcal{A}}^{m}$. Similar formulae hold for attractor $\mathcal{B}$.

The distance of a snapshot $\boldsymbol{u}$ to $\mathcal{B}$ is defined by the closest corresponding snapshot of $\mathcal{B}:$

$$
D(\boldsymbol{u}, \mathcal{B})=\min _{\substack{m=1, \ldots, M \\ x_{\mathcal{B}}^{m}=1}} D\left(\boldsymbol{u}, \boldsymbol{u}^{m}\right) .
$$

The average distance of attractor $\mathcal{A}$ to $\mathcal{B}$ is defined by

$$
D(\mathcal{A}, \mathcal{B})=\frac{1}{M_{A}} \sum_{\substack{m=1, \ldots, M \\ x_{\mathcal{A}}^{m}=1}} D\left(\boldsymbol{u}^{m}, \mathcal{B}\right) .
$$

This distance is not commutative. Suppose $\mathcal{A}$ has only one snapshot of $\mathcal{B}$ and $\mathcal{B}$ has many more elements. In this case, $D(\mathcal{A}, \mathcal{B})=0$ but $D(\mathcal{B}, \mathcal{A})>0$. Hence, we define a symmetrized version of this distance

$$
D_{\text {geom }}(\mathcal{A}, \mathcal{B}):=\frac{D(\mathcal{A}, \mathcal{B})+D(\mathcal{B}, \mathcal{A})}{2} .
$$

We refer to this quantity as Metric of Attractor Overlap (MAO). MAO has the properties of a metric for snapshot ensembles $\mathcal{A}$ and $\mathcal{B}$ and $\mathcal{C}$ since the defining properties can be shown for the generic case of pairwise different snapshots.

(1) Positive definiteness: $D_{\text {geom }}(\mathcal{A}, \mathcal{B}) \geqslant 0$ for all $\mathcal{A}, \mathcal{B}$ and

$$
\mathcal{A}=\mathcal{B} \quad \Leftrightarrow \quad D_{\text {geom }}(\mathcal{A}, \mathcal{B})=0 .
$$

(2) Symmetry: By definition (3.5),

$$
D_{\text {geom }}(\mathcal{A}, \mathcal{B})=D_{\text {geom }}(\mathcal{B}, \mathcal{A})
$$

(3) Triangle inequality:

$$
D_{\text {geom }}(\mathcal{A}, \mathcal{C}) \leqslant D_{\text {geom }}(\mathcal{A}, \mathcal{B})+D_{\text {geom }}(\mathcal{B}, \mathcal{C})
$$

To illustrate the physical implications of MAO, we consider several cases. If $\mathcal{A}$ and $\mathcal{B}$ only consist of velocity fields $\boldsymbol{u}$ and $\boldsymbol{v}$, respectively, their distance coincides with the Hilbert-space metric, $D(\mathcal{A}, \mathcal{B})=D(\boldsymbol{u}, \boldsymbol{v})$

Let $\mathcal{A}$ contain one velocity field, e.g., an unstable steady solution, and $\mathcal{B}$ contains many fields, e.g., snapshots of a stable periodic dynamics. Then $D(\mathcal{A}, \mathcal{B})$ is the smallest distance between the steady solution and the limit cycle, while $D(\mathcal{B}, \mathcal{A})$ represents the average distance between fixed point and limit cycle. This asymmetry makes sense. $D(\mathcal{A}, \mathcal{B})$ quantifies how well elements of $\mathcal{A}$ can be represented by elements of $\mathcal{B}$ on average. This measure is inherently non-communitative: A small set may be better represented by a rich set than the other way round. MAO is the average of both, i.e., an effective average distance between two attractors, not the minimal geometric distance between the closest elements of two sets.

Next, let us assume that both attractors arise from periodic dynamics defining limit cycles with the same origin and the same plane but with radii 10 and 11 . In this case, the distance approaches unity for sufficiently large amount of snapshots. Complete attractor 
overlap, $D_{\text {geom }}(\mathcal{A}, \mathcal{B})=0$, implies that $\mathcal{A}$ and $\mathcal{B}$ have the same snapshots. The probability of their occurrence may differ in both data.

In summary, MAO averages how well each snapshot of $\mathcal{A}$ is represented by the closest snapshot of $\mathcal{B}$ and vice versa. Note that some of the above statements need to be refined in case of identical snapshots. We shall not pause to do so.

\subsection{Proximity map}

In case of two attractors $\mathcal{A}$ and $\mathcal{B}$, the closeness can be characterized by MAO, i.e., a single number. The comparison of many attractors $\mathcal{A}^{l}, l=1, \ldots, L$ with $L \gg 1$ is more challenging. In this case, the complete snapshot set $\left\{\boldsymbol{u}^{m}\right\}_{m=1}^{M}$ comprises the elements of all $L$ attractors. The closeness of the attractors $\mathcal{A}^{l}$ may be visualized in a two-dimensional proximity map which preserves the metric of attractor overlap as good as possible (see figure 7 top, right). This task is performed by multi-dimensional scaling (Cox \& Cox 2000) presented in the following.

The relative distances between the attractors is expressed by the symmetric matrix

$$
D_{l n}:=D_{\text {geom }}\left(\mathcal{A}^{l}, \mathcal{A}^{n}\right), \quad l, n=1, \ldots, L .
$$

Let $\gamma^{l} \in \mathbb{R}^{2}$ be a two-dimensional feature vector associated with the $l$ th operating condition. The goal of a proximity map is to find a mapping $\mathcal{A} \mapsto \gamma$ such that the pointwise distances in the feature plane are preserved as good as possible,

$$
\sum_{l, n=1}^{L}\left(D_{l n}-\left\|\gamma^{l}-\gamma^{n}\right\|\right)^{2} \stackrel{!}{=} \min .
$$

At this point, the feature plane is indeterminate with respect to translation, rotation, and reflection. However, one can request that the center of the feature vector is at the origin, removing the translative degree of freedom. Moreover, the variances of the first feature coordinate can be maximized, removing the rotational degree of freedom. Now, only the indeterminacy with respect to reflection is left, like for POD modes.

The proximity map can easily be generalized for higher-dimensional feature spaces. Moreover, the proximity map requires only a distance matrix and can thus also be used for snapshots using the snapshot metric (3.1). Proximity maps have been presented for mixing layer and Ahmed body wake data (Kaiser et al. 2014) and for ensembles of control laws (Kaiser et al. 2017b; Duriez et al. 2016; Kaiser et al. 2017a).

\subsection{Cluster analysis}

Next, the $M$ snapshots $\boldsymbol{u}^{m}(\boldsymbol{x}), m=1, \ldots, M$, are coarse-grained into $K$ representative centroids $\boldsymbol{c}_{k}(\boldsymbol{x}), k=1, \ldots, K$. These centroids are chosen to minimize the total variance of the snapshots $\boldsymbol{u}^{m}$ with respect to the nearest centroid $\boldsymbol{c}_{k}$,

$$
V=\sum_{k=1}^{K} \sum_{\boldsymbol{u}^{m} \in \mathcal{C}_{k}} D^{2}\left(\boldsymbol{u}^{m}, \boldsymbol{c}_{k}\right) \stackrel{!}{=} \min .
$$

Each centroid $\boldsymbol{c}_{k}$ defines a cluster $\mathcal{C}_{k}$ containing all flow states $\boldsymbol{u}^{m}$ which are closer to $\boldsymbol{c}_{k}$ as compared to any other centroid $\boldsymbol{c}_{j}, j \neq k$. Thus, each snapshot $\boldsymbol{u}^{m}$ can be attributed to one cluster $\mathcal{C}_{k}$. This cluster affiliation is coded as characteristic function

$$
T_{k}^{m}:= \begin{cases}1, & \text { if } \boldsymbol{u}^{m} \in \mathcal{C}_{k}, \\ 0, & \text { otherwise. }\end{cases}
$$


The number of snapshots in the $k$ th cluster reads

$$
N_{k}=\sum_{m=1}^{M} T_{k}^{m}
$$

The centroids can also be expressed in terms of this characteristic function. It can be shown that they are the mean of all snapshots in the corresponding cluster,

$$
\boldsymbol{c}_{k}=\frac{1}{N_{k}} \sum_{\boldsymbol{u}^{m} \in \mathcal{C}_{k}} \boldsymbol{u}^{m}=\frac{1}{N_{k}} \sum_{m=1}^{M} T_{k}^{m} \boldsymbol{u}^{m} .
$$

Numerically, the optimization problem (3.8) for the centroids is solved using k-means clustering (Steinhaus 1956; MacQueen 1967; Loyd 1982) and k-means++ (Arthur \& Vassilvitskii 2007) for the initialization. Since k-means shows a dependence on the initial conditions, we run the corresponding MATLAB routine for 1000 initial conditions and select the one having the smallest variance. The iteration stops when convergence is reached, i.e., the characteristic function (3.9) does not change. The number of iterations is limited to 10,000 iterations.

For practical reasons, we perform a lossless POD decomposition prior to the clustering of $M$ snapshots into the mean flow $\boldsymbol{u}_{0}$ and $M-1$ modes $\boldsymbol{u}_{i}, i=1, \ldots, M-1$ (see $\S \mathrm{A}$ ). Let $\boldsymbol{u}=\boldsymbol{u}_{0}+\sum_{m=1}^{M-1} a_{i} \boldsymbol{u}_{i}$ be one snapshot and $\boldsymbol{v}=\boldsymbol{u}_{0}+\sum_{m=1}^{M-1} b_{i} \boldsymbol{u}_{i}$ another one. Then, $D^{2}(\boldsymbol{u}, \boldsymbol{v})=\sum_{m=1}^{M-1}\left(a_{i}-b_{i}\right)^{2}$. The right-hand side requires $3 M$ floating point operations while the computational load of the integral (3.1) scales with the number of grid points and is much larger even for the employed grids.

The clustering enables to characterize each operating condition by a corresponding probability distribution. Let $l=1, \ldots, L$ be the index of the $L$ operating conditions. Let $N_{k}^{l}$ be the number of snapshots of the $l$ th attractor data in the $k$ th cluster. Let $N^{l}=\sum_{k=1}^{K} N_{k}^{l}$ be the total number of snapshots of the $l$ th attractor data. Then, the probability that a snapshot belonging to the $l$ th operating condition lies in the $k$ th cluster reads

$$
P_{k}^{l}=\frac{N_{k}^{l}}{N^{l}}, \quad k=1, \ldots, K .
$$

In particular, the probability distribution is determined here as relative frequencies of cluster visits and fulfills the non-negativity, $P_{k}^{l} \geqslant 0$, and normalization condition, $\sum_{k=1}^{K} P_{k}^{l}=1$.

\subsection{Cluster-based analysis of the attractor overlap and dissimilarity}

In this section, we define a measure for the attractor similarity based on the centroid configuration. Each snapshot $\boldsymbol{u}^{m}$ is represented by its closest centroid $\boldsymbol{c}_{k}$. Let $\mathcal{A}$ and $\mathcal{B}$ be two sets of attractor data and $\boldsymbol{P}$ and $\boldsymbol{Q}$ be the corresponding cluster-based probability distributions. The snapshot-based metric is coarse-grained to the average distance of the centroids of attractor $\mathcal{A}$ and $\mathcal{B}$ :

$$
D_{\text {geom }}(\boldsymbol{P}, \boldsymbol{Q})=\frac{1}{2} \sum_{k=1}^{K}\left[P_{k} D\left(\boldsymbol{c}_{k}, \mathcal{B}^{c}\right)+Q_{k} D\left(\boldsymbol{c}_{k}, \mathcal{A}^{c}\right)\right] .
$$

Here $\mathcal{A}^{c}$ and $\mathcal{B}^{c}$ denote the centroid ensembles associated with attractors $\mathcal{A}$ and $\mathcal{B}$, respectively. This formula can be derived from (3.5) be replacing the snapshots $\boldsymbol{u}^{m}$ by the closest centroids $\boldsymbol{c}_{k}$ and taking into account the population size. The center row of figure 7 illustrates the approximative cluster-based analysis. Evidently, (3.13) is an approximation 
of (3.5) and is equivalent for the maximum cluster resolution $K=M$ of snapshots. In this case, each snapshot represents a centroid and the probability distributions associated with each cluster are unit vectors.

The main computational load for MAO comes from the distance matrix of the snapshots or centroids. The computational savings of the cluster-based measure (3.13) with respect to snapshot-based metric (3.5) scale with $(K / M)^{2}$. For the fluidic pinball study with $M=3500$ snapshots and $K=50$ clusters, this translates to the quite dramatic factor of $\approx 2 / 10000$. This saving does not include the operations for clustering. These can be large for a field-based clustering of $\S 3.4$ and small for an alternative clustering, e.g., based on few aerodynamic force components.

A lossless POD reduces the computational load of the distance matrix and of the clustering to a tiny fraction of the original cost. The main cost of POD originates from the correlation matrix which requires a similar amount of operations as the distance matrix. The advantage of POD preprocessing is the many post-processing options at negligible cost.

As a side note, a commonly used metric for probability distributions is the JensenShannon distance (see appendix $\S \mathrm{B}$ ). This distance measures probability differences on shared clusters but is blind to the geometric distances of disjoint clusters. In the previous example of concentric co-planar circles, the metric is the same for all non-identical radii.

\section{Comparison of fluidic pinball attractors with different control laws}

The comparison of fluidic pinball attractors is performed following the proposed methodology of the previous section. For simplicity and consistency, we follow the coarsegrained data comparison displayed in the center and bottom row of figure 7. Firstly ( $\S 4.1$, the cluster analysis is performed. Sections $\S 4.2$ and $\S 4.3$ present the metric of attractor overlap and associated proximity maps. It should be noted that the analysis is only based on converged post-transient data while the shown temporal dynamics includes also the actuation transients.

\subsection{Cluster analysis}

Figure 8 illustrates 50 centroids distilled from $7 \times 500$ post-transient snapshots. The cluster affiliation as a function of time is shown in figure 9. This affiliation illustrates the role of each centroid (figure 8) in the posttransient behaviors of control laws $I-$ $V I I$ of Table 1. Clusters 1-5 describe unforced vortex shedding of phase $I$ (see figure 8. We emphasize that the centroids are only meant to resolve converged attractor data and that the presentation of the transient dynamics shall only indicate the transient times. The stabilized boat tailing of phase $I I$ is described by a single centroid $k=6$. Base bleed (phase $I I I$ ) is resolved by the new centroids 7-20, indicating that base bleed leads to significantly different vortex shedding structures. High-frequency (phase $I V$ ) and low-frequency forcing (phase $V$ ) can be resolved with the centroids of unforced vortex shedding and of base-bleed actuation. Low-frequency forcing leads to a wider wake, like base bleed, and has more overlap with base-bleed dynamics. The new centroids 34-41 resolve the positive Magnus effect (phase VI) while centroids 42-50 the negative Magnus effect (phase VII). As physical intuition suggests, the strong deflected wake has no overlap with forced states which are symmetric or statistically symmetric.

Figure 10 displays the population of each cluster by the seven different dynamics. Probabilities below $1 \%$ are kept white. The probability distributions for each attractor are far from being uniform. This behavior is different from single attractor cluster analyses, where all clusters are generally populated (Kaiser et al. 2014). In contrast, considering 


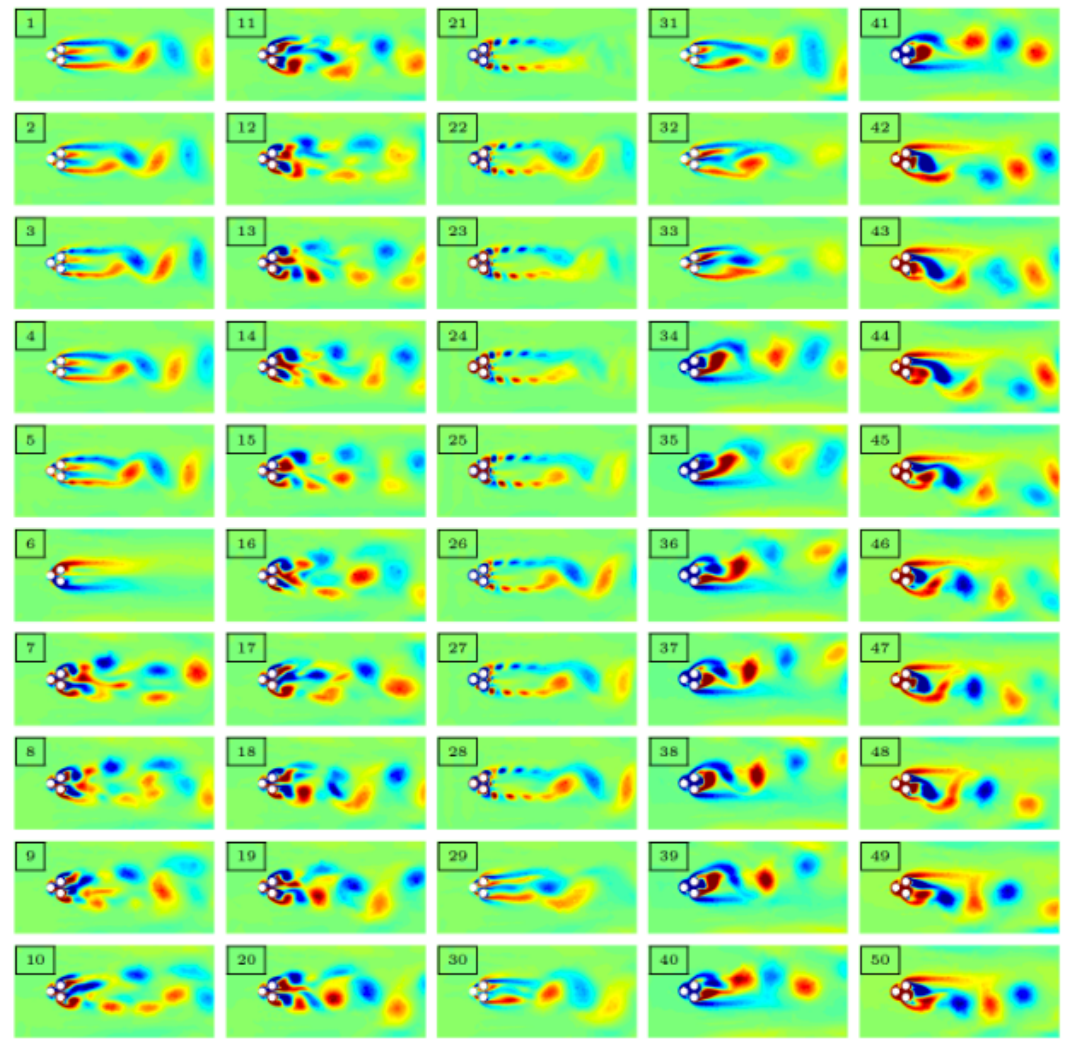

Figure 8. Cluster centroids of the fluidic pinball simulation. The centroids are sorted in order of appearance neglecting the first 50 time units (transients) of each phase. The visualization depicts the vorticity distribution: green, red and blue represent vanishing, positive and negative values, respectively.

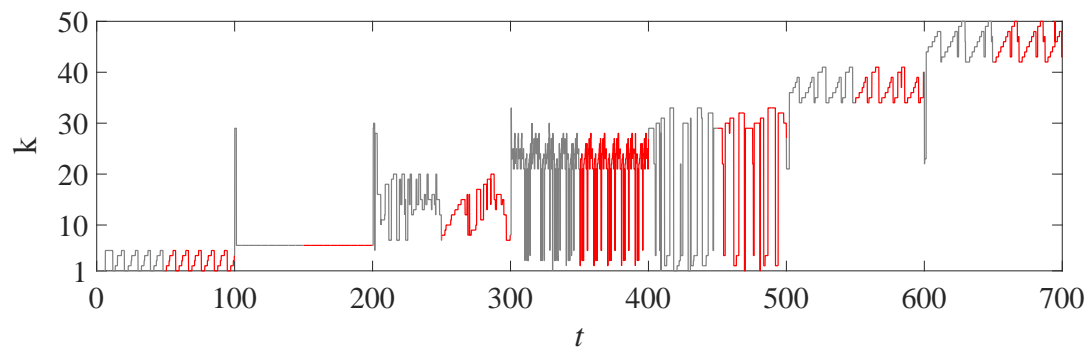

Figure 9. Cluster affiliation $k$ of the fluidic pinball simulation as a function of time $t$. The applicable control laws are described in table 1 and the corresponding centroid $\boldsymbol{c}_{k}$ is depicted in figure 8. By construction, new cluster indices are a monotonously increasing function of time, neglecting the initial actuation transients. Gray curve sections correspond to transients while red sections mark the posttransient data taken for the analysis.

the whole simulation results in the total probability $P_{k}=P_{k}^{1}+\ldots+P_{k}^{7}$ which is nonzero for any cluster $k$. Note that one cluster may be populated by different attractor dynamics. 


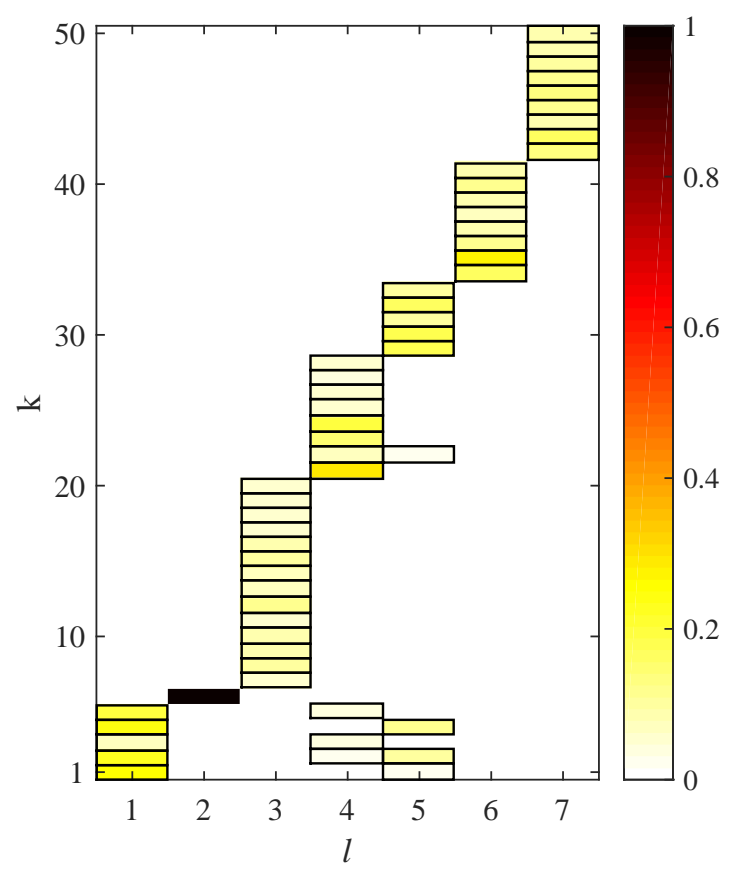

Figure 10. Population probabilities of the clusters for the seven fluidic pinball phases (table 1 ). The abscissa denotes the phase $l$ and the ordinate the cluster index $k$. Each box represents a non-vanishing probability. The yellow to red tones indicate the population probability $P_{k}^{l}$ from (3.12). Probabilities below $1 \%$ are kept white. Note that steady boat tailing $(l=2)$ is represented by a single centroid.

\subsection{Metric of attractor overlap}

The metric of attractor overlap for the seven operating conditions is displayed in figure 11. The positive (semi)definiteness implies the vanishing elements in the diagonal and non-negative values elsewhere. The symmetry condition leads to the corresponding symmetry of the matrix. The values of the metric are based on the Hilbert space norm (3.5) and an averaging process over the two attractors (3.1). Hence, the fluctuation level, i.e., twice the fluctuation energy $2 \mathrm{TKE}$, represents a natural reference value for the square of the metric. Values which are at least one order of magnitude smaller correspond to similar attractors. For the unforced fluidic pinball configuration, the fluctuation level is $2 \mathrm{TKE}=17.80$. Hence, a MAO value of $\sqrt{2 \mathrm{TKE}}=4.22$ can be considered as reference scale for closeness.

Natural vortex shedding $(l=1)$ is seen to be close to high- and low-frequency forcing $(l=4,5)$, as the metric is a tiny value of the maximum (small circles). This closeness is corroborated by the probability distributions 10: the three states share joint clusters. In contrast, the wake stabilized with boat tailing shares no centroids with the other six operating conditions and has a large MAO distance to them. The values are comparable or exceed the reference scale. Similarly, the positive and negative Magnus effect $(l=6,7)$ have a large distance to each other, as expected from the opposite deflections of the wake in figure 3 and the empty overlap of both attractors, i.e., no shared clusters in figure 10. 


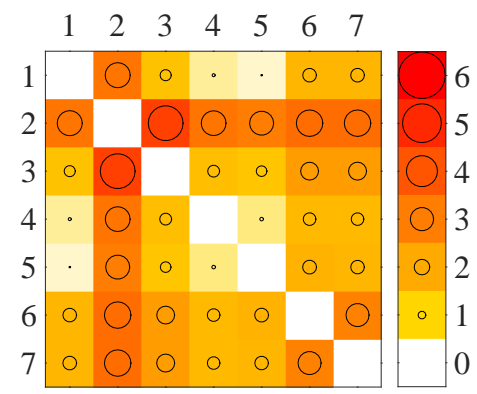

FiguRE 11. Metric of attractor overlap (3.13) for the seven control laws of the fluidic pinball (table 1). The value of $D_{\text {geom }}\left(\boldsymbol{P}^{l}, \boldsymbol{P}^{n}\right)$ is shown in the $l$ th row and $n$th column as color code from white to red and by the size of the circle as illustrated by the caption on the right.

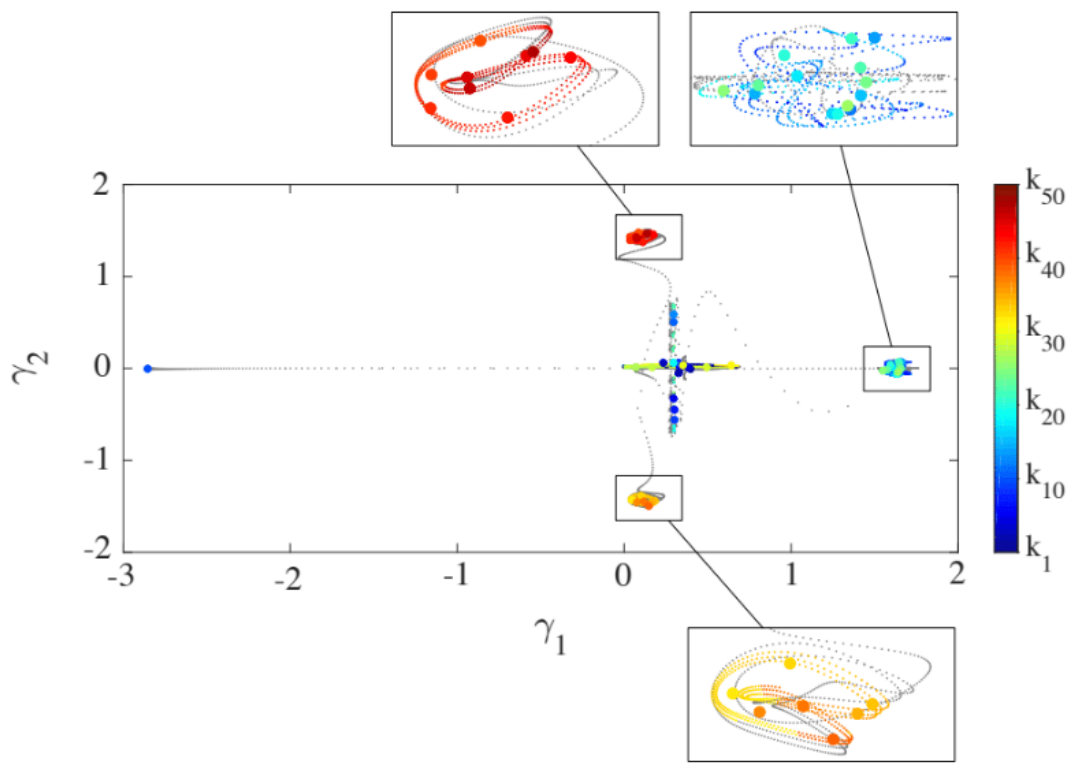

FiguRE 12. Proximity map of the fluidic pinball showing the centroids (large circles) together with snapshots (small circles). Snapshots from transients are displayed as gray dots, while the data for the analysis is color-coded by cluster affiliation.

\subsection{Proximity map}

Figure 12 displays all centroids and all snapshots in a proximity map following $\S 3.3$. Phase $I-V$ attractors are located on the $\gamma_{2}=0$ line. These flows are statistically symmetric with respect to the $x$-axis and have, correspondingly, vanishing average lift. Phase VI and VII correspond to positive and negative Magnus effects associated with negative and positive average lift, respectively. These phases are mirror-symmetrically located in the lower and upper region. The second feature coordinate $\gamma_{2}$ is clearly related to averaged lift. The lift values of all phases are displayed in figure 3 .

The boat-tailed (phase $I I$ ) and base-bleed dynamics (phase $I I I$ ) represent the minimal and maximum drag states of considered dynamics, referring again to figure 3 . These attractors are on the leftmost and rightmost sides near the $\gamma_{1}$-axis, respectively. The other attractors have similar drag and have $\gamma_{1} \approx 0$. Summarizing, the first feature coordinate is strongly correlated to drag. It should be noted that the automatically 
(a)

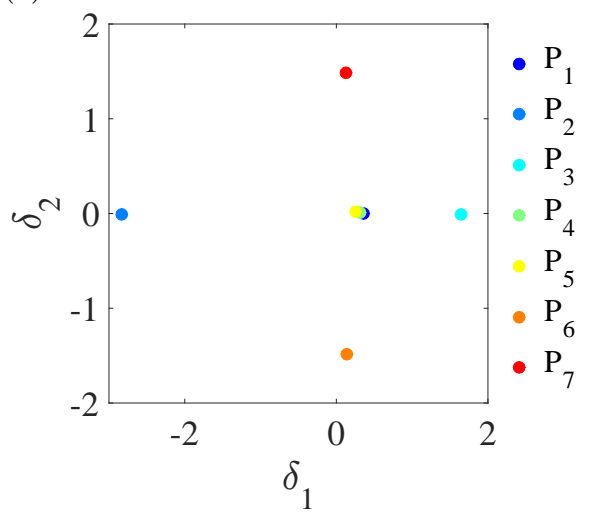

(b)

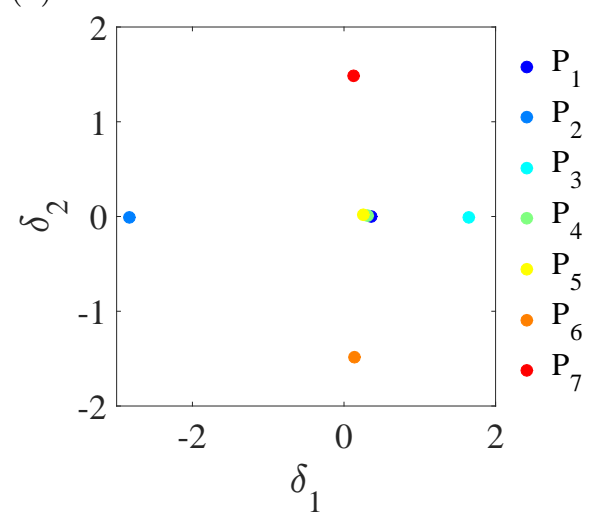

FIgURE 13. Attractor proximity map of the fluidic pinball: (a) based on the snapshot-based MAO (3.5) and (b) on a cluster-based MAO (3.13) with $K=50$ clusters. The points $P_{1} \ldots P_{7}$ correspond to the seven control laws in table 1.

determined feature coordinates are strongly linked to aerodynamic forces although the forces did not enter the multi-dimensional scaling analysis.

The proximity map for the attractors based on MAO is depicted in figure 13, both, for the snapshot-based definition (a) and the cluster-based estimate (b). By construction, the distances between the feature vectors of the seven attractors are similar the MAO metric values displayed in figure 11. are similar to Both maps are virtually indistinguishable, thus showing the accuracy of the cluster-based approximation. Varying the cluster resolution in a wide range $K \in\{25,50,100\}$ corroborates the robustness of the cluster-based MAO: For all values, the proximity maps are an excellent approximation of the snapshot-based version.

The feature coordinate $\delta_{1}$ correlates with the drag and $\delta_{2}$ with the average lift-like in figure 12. Even the numerical values of the feature coordinates associated with the same operating conditions are similar. and the attractor proximity map is an aggregate of the snapshots and centroids. This quantitative similarity indicates the robustness of the multi-dimensional scaling for the construction of proximity maps.

\section{Comparison of turbulent boundary layers at different wall actuation}

We analyze the three-dimensional flow data generated by 38 simulations of the actuated turbulent boundary layer using the cluster-based MAO methodology. In $\S 5.1$ we present the cluster analysis of the 38 attractors. In $\S 5.2$ and $\S 5.3$ the MAO analysis and proximity maps for snapshots, centroids and attractors are illustrated.

\subsection{Cluster analysis}

Figure 14 displays the probability distribution of each attractor to pass through a given cluster. Again, probabilities below 1\% are kept white. All attractors have pair-wise different cluster compositions. The unforced reference $l=1$ occupies the first five clusters. The attractors $l=2, \ldots, 9$ corresponding to a spanwise wavelength $\lambda=200$ populate clusters $k=5, \ldots, 16$. The attractor $l=7$ with low actuation amplitude shares four of five clusters with the unforced flow and populates one new cluster $k=14$. The simulations with a spanwise wavelength $\lambda^{+}=500(l=10, \ldots, 17)$ pass through the new clusters 


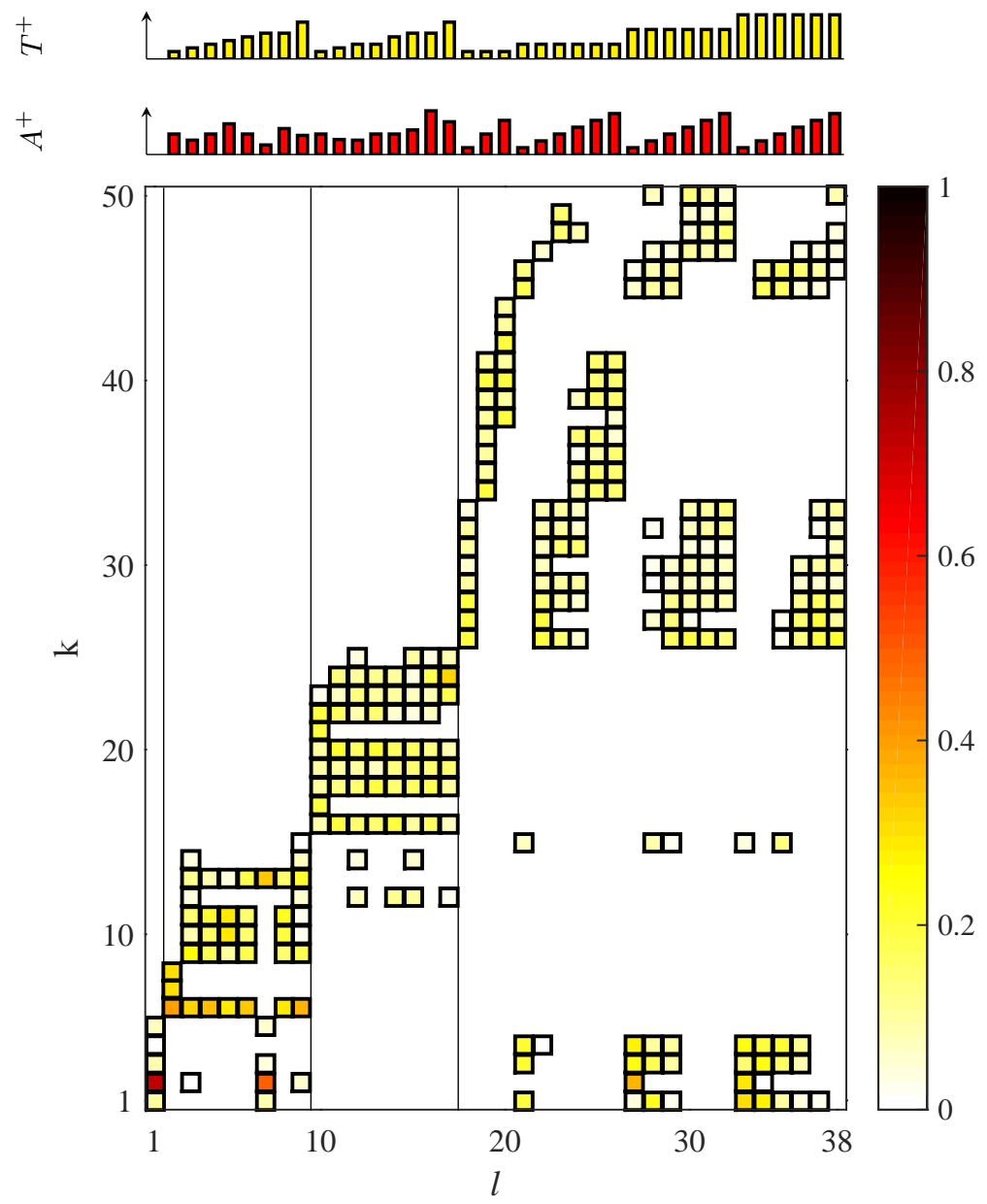

Figure 14. Population probabilities of the clusters for the 38 turbulent boundary layer simulations (see table 3). The abscissa denotes the data set $l$ and the ordinate the cluster index $k$. Each box represents a non-vanishing probability. The yellow to red tones indicate the population probability or relative frequency $P_{k}^{l}$ from (3.12). Probabilities below $1 \%$ are kept white. The actuation wavelength is indicated by the three vertical line separating simulations with no actuation, $\lambda^{+}=200, \lambda^{+}=500$ and $\lambda^{+}=1000$ from left to right. The actuation amplitude $A^{+}$and time $T^{+}$are shown in the top caption.

$k=18, \ldots, 26$. These attractors have no overlap with the unforced reference and share few states with the previous group at low-wavelength actuation. The long-wavelength actuation $\left(\lambda^{+}=1000\right)$ populates the new clusters $l=27, \ldots, 50$. This group shares only one cluster $(l=16)$ with the medium-wavelength group but may have significant overlap with the unforced reference. This is particularly true for low-amplitude $\left(A^{+}=10\right)$ actuations at $l=21,27$, and 33. It should be noted that the MAO methodology displays the overlap between attractors in a computer- and human-interpretable form.

\subsection{Metric of attractor overlap}

Figure 15 displays the geometric distance between the attractors based on MAO. Evidently the diagonal vanishes by definition. In complete analogy to the fluidic pinball, the reference for a large scale can be taken to be the fluctuation amplitude $\sqrt{2 \mathrm{TKE}}=$ 


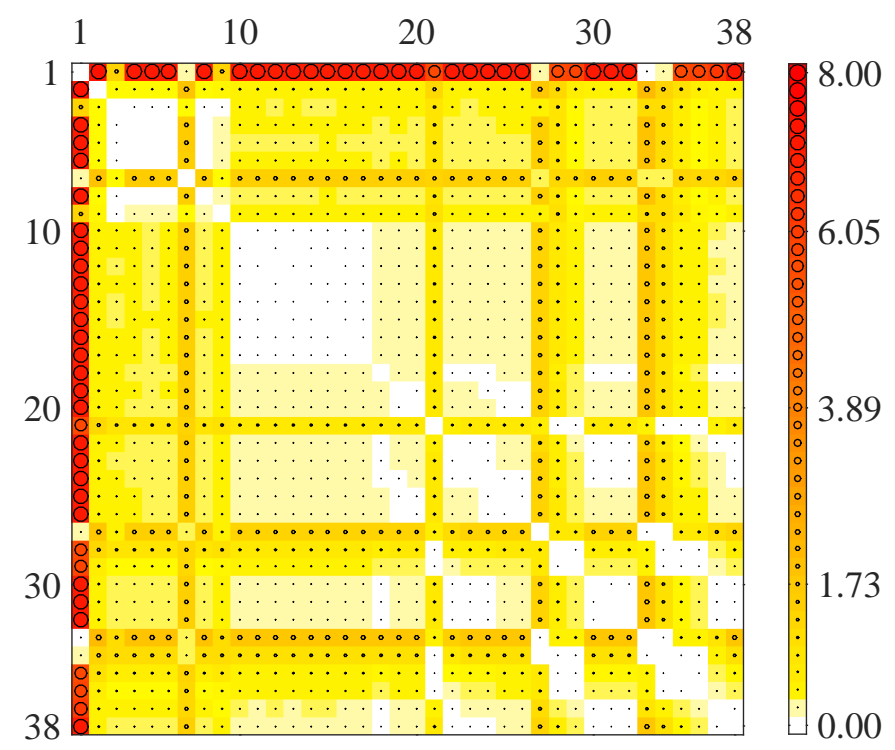

FiguRE 15. Metric of attractor overlap (3.13) of the turbulent boundary layer with 38 control laws (see table 3). The visualization is analogous to figure 11 .

0.8767 of the unforced flow $(A=0)$, noting that actuation may increase this amplitude by one order of magnitude. Most of metric values are in this range or lower.

The unforced reference $l=1$ is seen to be very different from most other attractors. Attractors 2 to 6 are very close to each other, which can be expected because of their same actuation wavelength $\lambda^{+}=200$. Again, attractors 10 to 17 corresponding to wavelength $\lambda^{+}=500$ display significant similarity, i.e. a low MAO. The group of actuated cases with large wavelength $\lambda^{+}=1000$ show less similarity which is consistent with the discussed overlap from the previous figure. Attractors 7, 21, 27, and 33 have low actuation amplitude, share similar characteristics with the unforced reference as may be expected.

\subsection{Proximity map}

Figure 16 visualizes the distance between the centroids and snapshots. Actuations at low wavelengths (small $k$ ) are close to the origin while large wavelength actuation (large $k$ ) may populate circular regions in the periphery. A closer analysis reveals that $\gamma_{1}, \gamma_{2}$ correspond to the first POD mode amplitudes $a_{1}, a_{2}$ computed from all snapshot data together. These POD mode amplitudes are nearly periodic, particularly for large actuation amplitudes.

Figure 17 displays the proximity map of the attractors based on MAO. From Figure 17 (a) the first feature coordinate $\delta_{1}$ is linked to the drag reduction for the attractors: For $\delta_{1}<0$ actuation has little effect on drag but for $\delta_{1} \geqslant 0$ these changes are significantpositive for $\delta_{2}>0$ and negative for $\delta_{2}<0$. From Figure 17 (b), $\delta_{2}$ correlates with the wavelength of the traveling wave. The large wavelengths are located in the top half of the plot. As seen in the fluidic pinball, the MAO-based attractor proximity map extracts the aerodynamics and actuation properties without directly including information about them. 


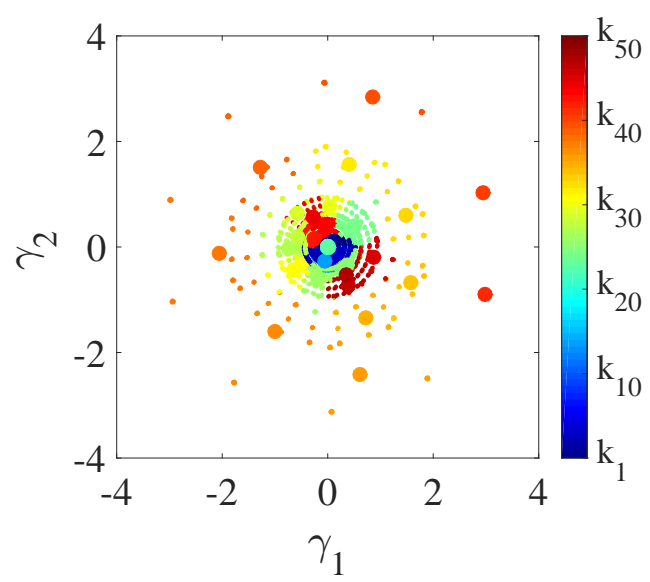

FiguRE 16. Proximity map of the actuated turbulent boundary layer. The figure displays the the centroids (large circles) together with snapshots (small circles). The data for the analysis is color-coded by cluster affiliation.

(a)

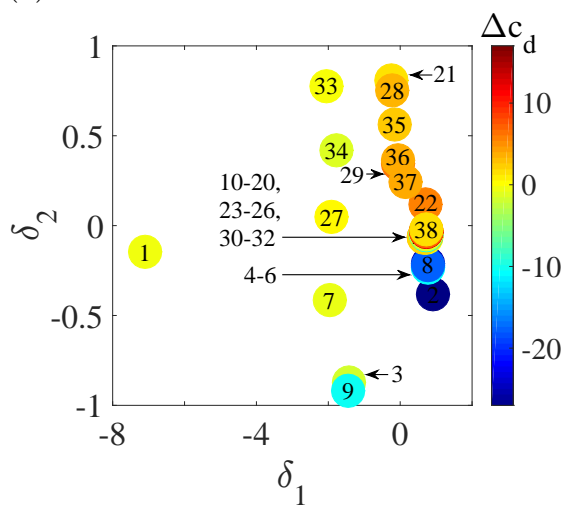

(b)

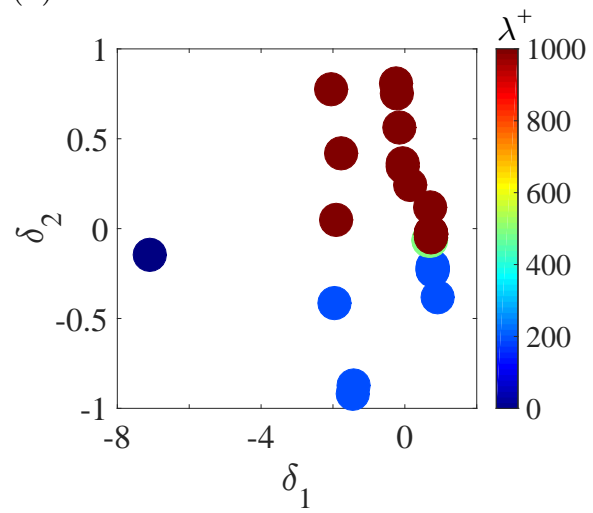

FigURE 17. Attractor proximity map of the turbulent boundary layer simulations (see table 3): (a) color-coded with relative drag reduction $\Delta c_{D}$; (b) color-coded with wavelength $\lambda^{+}$. The actuation parameters of each symbol is indicated in subfigure (a) by a number corresponding to the index from table 3 .

\section{Conclusions and outlook}

We have proposed a novel quantitative measure, termed Metric of Attractor Overlap (MAO), for the similarity of attractor data. This measure generalizes the Hilbert-space metric for two velocity fields to a metric between two sets with snapshot data. A refined comparison analysis includes proximity maps and coarse-graining with clustering.

Our proposed comparison methodology has five discriminating features. Firstly, the difference between two attractors is quantified in a single, non-negative number encapsulating all resolved coherent structures. Secondly, the cluster probability distribution of each attractor identifies shared and disjoint flow states via a manageable number of centroids. Thirdly, the metric of attractor overlap enables the automatic creation of a proximity map from a myriad of attractors. Neighboring feature points represent attractors with similar flow states, largely separated points indicate attractors with different physics. Fourthly, the approach is compatible with statistical post-processing: 
The centroids and their probabilities define the mean flow and - in principle - any higherorder moment, like Reynolds stress or fluctuation energy. Even POD modes can be derived from the centroids. Finally, the comparison methodology can be automatized and has little subjective bias. One does not need to decide in advance on a cost function, such as drag power, a frequency filter, such as low-pass filter, important flow features, such as vortices, just to name a few.

The metric of attractor overlap is only based on two decisions. First, one needs to choose the flow state space, e.g. the velocity field in a domain $\Omega$. Such a decision is unavoidable for any metric. Second, a metric for two flow states is needed. A $L^{2}(\Omega)$ Hilbert space norm appears as a natural candidate. For the optional coarse-graining, the number of clusters or, synonymously, the typical size of a cluster needs to be chosen. The coarse-grained metric is found to quickly converge to the snapshot-based one with increasing number of clusters.

The comparison methodology has been applied to the fluidic pinball, the flow around a cluster of three rotating cylinders. The considered attractors include the unforced reference, aerodynamic boat tailing, base bleed, symmetric high-frequency forcing, symmetric low frequency forcing and a Magnus effect deflecting the wake to both sides. The metric of attractor overlap confirms physical inspection of the seven configurations. First, boat tailing with complete wake suppression differs strongly from all other states. Second, both Magnus effects also strongly differ from all other states. Third, unforced vortex shedding and wakes manipulated by base bleed as well as high-frequency and low-frequency forcing have a significant overlap of shared clusters, i.e. are similar.

Particularly noteworthy are the proximity maps of the snapshots and the cluster-based attractors. The trajectory through all seven configurations displays the dynamics very clearly (see figure 3). Surprisingly, the first and second feature coordinates resemble the drag and lift respectively (see figure 13). The features of the snapshots show the reduced drag by boat tailing (left) and the increased drag by base bleed (right) and significant average lift by both Magnus forcing (top and bottom). The proximity map of the attractor visually elucidates the discussed neighborhood relations in the first three feature coordinates.

The second application of MAO is an open-loop drag reduction study of a turbulent boundary layer. The operating conditions include the unforced reference and 37 actuations with spanwise traveling waves at different amplitudes and frequencies. The spanwise wavelengths include a small $\left(\lambda^{+}=200\right)$, medium $\left(\lambda^{+}=500\right)$, and large value $\left(\lambda^{+}=1000\right)$. The computational and observation domain are defined by the largest actuation wavelength. The MAO analysis features several highlights. First, the low amplitude actuations share centroids with the unforced reference in agreement with physical intuition. Second, actuations with the same spanwise wavelength show significant overlap. Third, the feature map of the snapshots show low amplitude states near the center and large amplitude actuations populate outer circular regions. The feature coordinates are well aligned with POD mode amplitudes of the phase-averaged flow using actuation as a clockwork. Finally, the first feature coordinate of the attractor proximity map correlates well with the drag reduction - like for the fluidic pinball — while the second coordinate depends on the spanwise actuation wavelength.

The observed correlation between first feature coordinate and drag for two independent configurations is surprising as the input data does not contain information about drag. In hindsight, this behavior may be explained by the strong correlation between the timeaveraged flow and the drag - both, for the wake and for the boundary layer.

Evidently, the presented MAO comparison encourages numerous other applications, like a comparison between computed and experimental velocity fields and a comparison 
between flow behavior under different actuations, e.g. periodic forcing or machine learning control studies (Duriez et al. 2016). The analysis may also be applied to sensor data, e.g. a hot-wire rig, instead of velocity fields.

Future research may significantly extend the MAO methodology. The employed Hilbert space norm is a good initial choice but highly sensitive to small mode deformations (Noack 2016). A small change in wavenumber gives rise to unphysically large differences in the norm. Force-related feature vectors and manifold learning may be one remedy (Loiseau et al. 2018). Rigorous physics-based criteria for the cluster numbers need to be advanced. The comparison may also be targeted towards single- or multi-objective goals by generalizing the snapshot metric. So far, the comparison only addressed ergodic properties of the attractor, and the snapshot data only needed to be statistically representative and not time-resolved. The temporal dynamics of different attractors may be compared using time-resolved snapshots and corresponding Markov models (Kaiser et al. 2014).

Summarizing, a rational automated comparison method has been proposed which holds significant promise for future data assessments. The authors are actively pushing this direction.

\section{Acknowledgments}

This work is supported by a public grant overseen by the French National Research Agency (ANR) as part of the "Investissement dAvenir" program, through the "iCODE Institute project" funded by the IDEX Paris-Saclay, ANR-11-IDEX-0003-02, by the ANR grants "ACTIV_ROAD" and "FlowCon", by the Polish National Center for Research and Development under the Grant No. PBS3/B9/34/2015, and by the Bernd Noack Cybernetics Foundation. Additional funds are provided by the German Research Foundation (DFG) under grant numbers SE 2504/2-1 and SCHR 309/68-1. Computing resources were provided by the High Performance Computing Center Stuttgart (HLRS) and by the Jülich Supercomputing Center (JSC) within a large-scale project of the Gauss Center for Supercomputing (GSC).

EK gratefully acknowledges support by the "Washington Research Foundation Fund for Innovation in Data-Intensive Discovery" and a Data Science Environments project award from the Gordon and Betty Moore Foundation (Award \#2013-10-29) and the Alfred P. Sloan Foundation (Award \#3835) to the University of Washington eScience Institute.

We appreciate valuable stimulating discussions with Steven Brunton, Nathan Kutz, Themis Sapsis, and the French-German-Canadian-American pinball team: Guy Yoslan Cornejo-Maceda, Nan Deng, François Lussgeeyran, Robert Martinuzzi, Cedric Raibaudo, Romain Rolland and Luc Pastur.

\section{Appendix A. Proper orthogonal decomposition versus clustering}

The cluster analysis is compared with the proper orthogonal decomposition (POD) from the same data in the same observation domain. Each snapshot is expanded in terms of the mean flow $\boldsymbol{u}_{0}(\boldsymbol{x})$ of all post-transient attractor data and $N$ POD modes $\boldsymbol{u}_{i}(\boldsymbol{x})$, $i=1, \ldots, N$ and their corresponding amplitudes $a_{i}(t), i=1, \ldots, N$ :

$$
\boldsymbol{u}(\boldsymbol{x}, t) \approx \boldsymbol{u}_{0}(\boldsymbol{x})+\sum_{i=1}^{N} a_{i}(t) \boldsymbol{u}_{i}(\boldsymbol{x})
$$



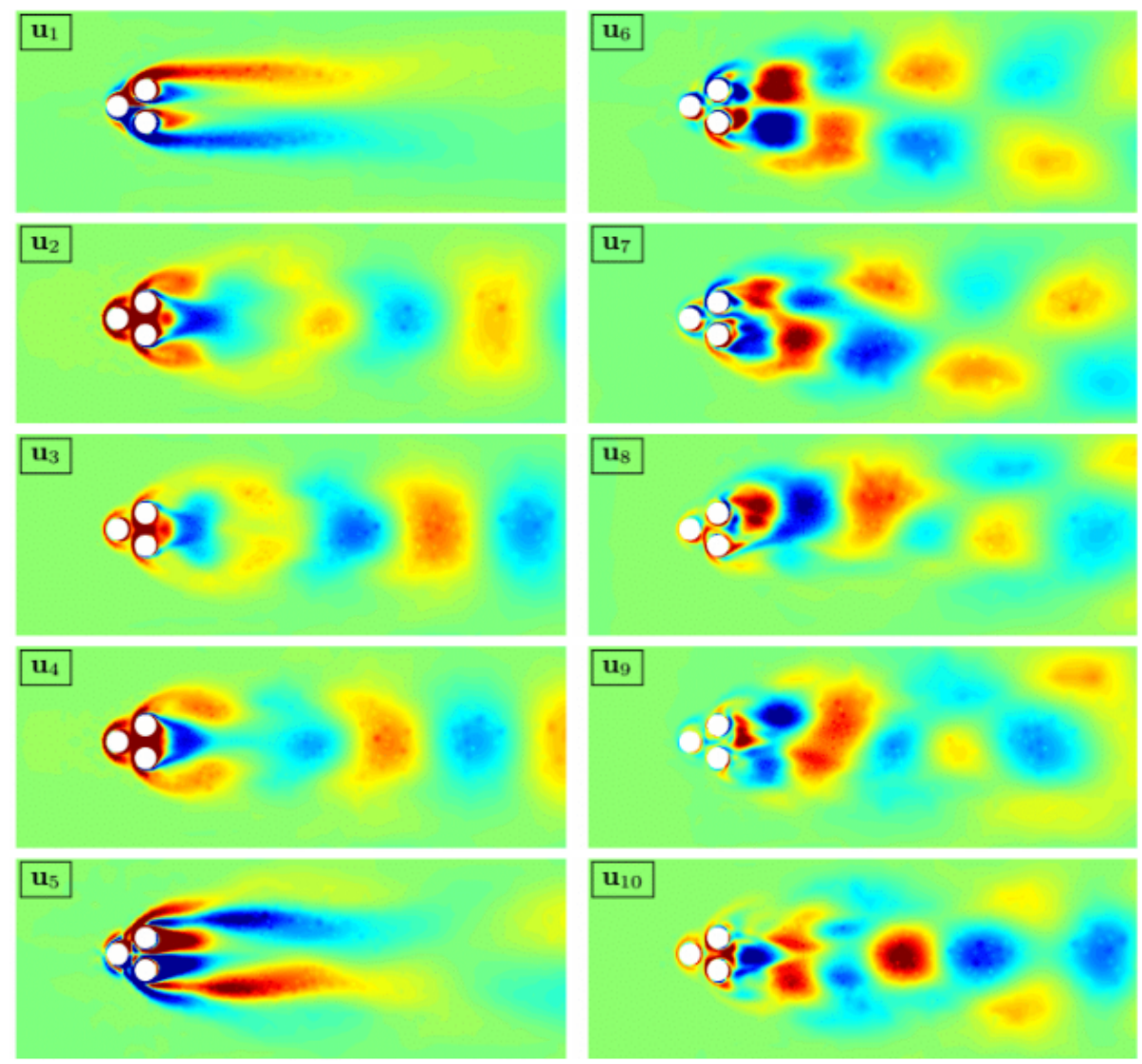

FIGURE 18. POD modes $\boldsymbol{u}_{i}(\boldsymbol{x}), i=1, \ldots, 10$.

Figures 18 and 19 display the first ten POD modes and their amplitudes, respectively. Mode 1 resolves a base-flow deformation, like a shift mode (Noack et al. 2003). Mode 5 represents a symmetric near-field modulation. The other modes have more oscillatory structures. We shall not pause to hypothesize about the physical meaning of these modes. Modes with cleaner frequency content yet near the optimal residual could, for instance, be constructed with recursive DMD (Noack et al. 2016). The cluster analysis resulting in the snapshot cluster affiliation (Fig. 9) and centroids (Fig. 8) is physically easier to interpret than the POD.

It may be noted that POD also allows for another metric of attractor overlap. Let $p(\boldsymbol{a})$ be the probability distribution associated with one attractor and $q(\boldsymbol{a})$ the distribution of another one. Then, the continuous version of the Jensen-Shannon distance (B 4) can be applied. An advantage is that the snapshot distance (3.1) is reflected in the construction of the metric. A disadvantage is the need for approximations to construct a continuous probability distribution from a finite number of snapshots.

\section{Appendix B. Jensen-Shannon distance}

Probability distributions can easily be compared using information measures. Let $\boldsymbol{Q}=\left[Q_{1}, \ldots, Q_{K}\right]$ be a reference probability distribution and $\boldsymbol{P}=\left[P_{1}, \ldots, P_{K}\right]$ a new measured one. Then, the information gained from $\boldsymbol{P}$ with respect to the reference $\boldsymbol{Q}$ is 

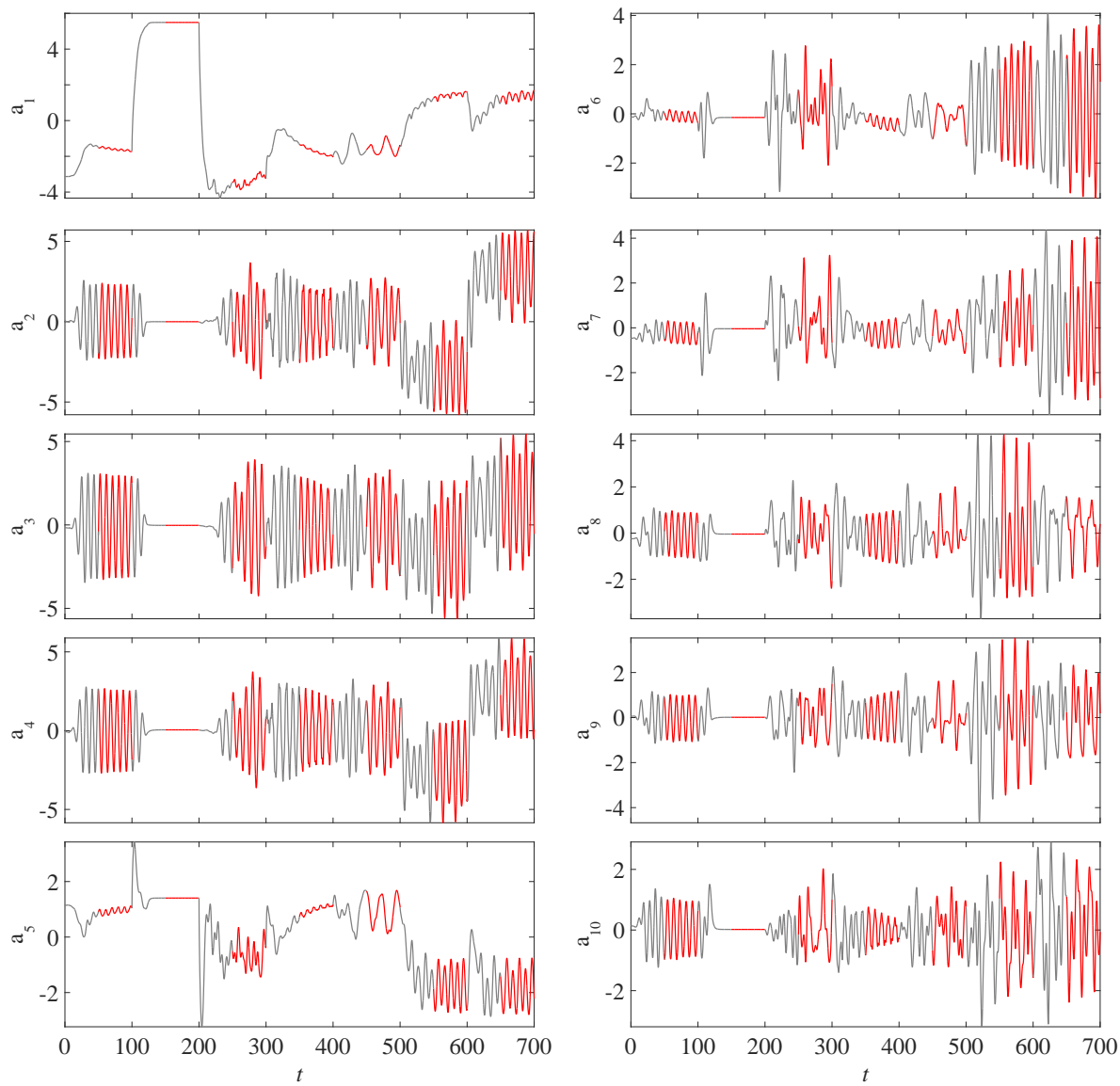

Figure 19. POD amplitudes $a_{i}(t), i=1, \ldots, 10$. Snapshot sequences after the decay of the transients are employed for the analysis (colored in red).

quantified by the Kullback-Leibler divergence (Kullback \& Leibler 1951; Kullback 1959), also called relative entropy,

$$
D_{K L}(\boldsymbol{P} \| \boldsymbol{Q})=\sum_{k=1}^{K} P_{k} \ln \left[\frac{P_{k}}{Q_{k}}\right] .
$$

Identical probability distributions $\boldsymbol{P}=\boldsymbol{Q}$ give rise to a vanishing Kullback-Leibler divergence. Different distributions yield a positive value. If $P_{k}=0$, the term $P_{k} \ln P_{k} / Q_{k}$ is interpreted as zero because $\lim _{x \rightarrow 0} x \ln x=0$. Strictly speaking, the Kullback-Leibler divergence is not defined in case there exists a $k$ for which $Q_{k}=0$ and $P_{k}>0$. One could follow a common practice of smoothing using the absolute discounting method by replacing $Q_{k}$ by a small value, e.g. $\epsilon=0.001$.

The Kullback-Leibler divergence is not symmetric, i.e. $D_{K L}(\boldsymbol{P} \| \boldsymbol{Q}) \not \equiv D_{K L}(\boldsymbol{Q} \| \boldsymbol{P})$. Hence, it cannot serve as a metric. The probability distributions $\boldsymbol{P}$ and $\boldsymbol{Q}$ may be compared with the Jensen-Shannon divergence which is defined as symmetrized and smoothened Kullback-Leibler divergence:

$$
J S D(\boldsymbol{P}, \boldsymbol{Q})=\frac{1}{2}\left[D_{K L}(\boldsymbol{P} \| \boldsymbol{M})+D_{K L}(\boldsymbol{Q} \| \boldsymbol{M})\right], \quad \text { where } \quad \boldsymbol{M}=\frac{1}{2}[\boldsymbol{P}+\boldsymbol{Q}] .
$$


(a)

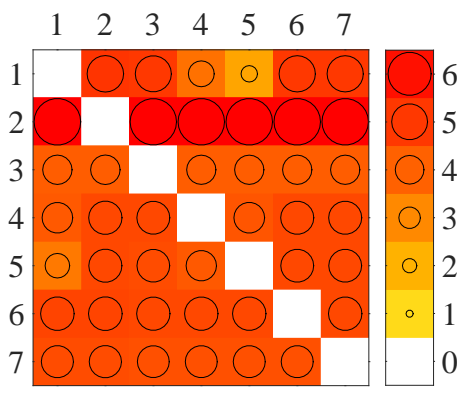

(b)

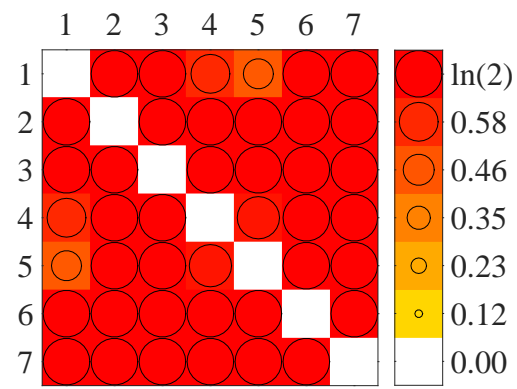

Figure 20. Distances between attractors: (a) Kullback-Leibler divergence and (b) Jensen-Shannon divergence based on the cluster probability distribution for each of the seven control phases. The row and column of $D_{K L}(P \| Q)$ corresponds to the first $(\boldsymbol{P})$ and second argument $(\boldsymbol{Q})$. The value of the divergence is color-coded and indicated by the size of the circle (see the corresponding caption).

Equivalently,

$$
J S D(\boldsymbol{P}, \boldsymbol{Q})=\frac{1}{2} \sum_{k=1}^{K}\left(P_{k} \ln \left[\frac{2 P_{k}}{P_{k}+Q_{k}}\right]+Q_{k} \ln \left[\frac{2 Q_{k}}{P_{k}+Q_{k}}\right]\right) .
$$

The summation term is interpreted as zero if $P_{k}=Q_{k}=0$ or if the numerator of the logarithm argument vanishes. Note that we don't need the $\epsilon$ threshold of the KullbackLeibler entropy anymore.

The square root of the Jensen-Shannon divergence has all properties of a metric (Endres \& Schindelin 2003) and is referred to as Jensen-Shannon distance,

$$
D_{J S}(\boldsymbol{P}, \boldsymbol{Q})=\sqrt{J S D(\boldsymbol{P}, \boldsymbol{Q})} .
$$

This distance (B 4) defines an entropic metric of attractor overlap between two attractor data represented by the cluster distributions $\boldsymbol{P}$ and $\boldsymbol{Q}$.

Figure 20 displays the Kullback-Leibler and Jensen-Shannon divergences between the seven fluidic pinball attractors. The diagonal entries vanish by definition. Unforced vortex shedding is seen to be similar to high- and low-frequency forcing, as was indicated already by the many shared clusters in figure 9 . The difference between unforced shedding, basebleed dynamics, and both Magnus effects is larger as they share no joint clusters. The stabilized boat tailing with a single cluster is seen to be very different from all other phases with no cluster overlap. The Kullback-Leibler divergence is, as expected, not symmetric. For instance. the divergence of boat-tailing with respect to base-bleed is larger than the other way round. It should be noted that the Jensen-Shannon distance is typically $\ln (2)$ (or unity if the base 2 logarithm is used) corresponding to no shared clusters.

The Jensen-Shannon distance is a natural metric for probability distributions. Yet, it is blind to the geometric location of the centroids. Let us assume the first attractor data populates only cluster $1, P_{i}=\delta_{i, 1}$, and the second data occupies only cluster 2 , $Q_{i}=\delta_{i, 2}$. In this case, $J S D(\boldsymbol{P}, \boldsymbol{Q})=\ln 2$, regardless of whether the centroids are very close or very far from each other. This property makes the Jensen-Shannon distance strongly dependent on the number of clusters or, equivalently, on the typical size of the clusters. For the minimum number of clusters, $K=1$, we have obtained the trivial result $P_{1}=Q_{1}=1$ and $J S D=0$. For the maximum number of clusters, each snapshot represents one centroid and defines one cluster. In the generic case of different snapshots, the Jensen-Shannon distance is also $\ln 2$ independent of the geometric location of the 
snapshots. Only a few of the seven fluidic pinball phases share joint clusters and the proximity maps based on (B 4) provides limited physical insight. The metric may be far more meaningful in other cases in which most attractors have significant overlap.

\section{REFERENCES}

Alkishriwi, N, Meinke, M \& SchröDER, W 2006 A large-eddy simulation method for low mach number flows using preconditioning and multigrid. Comp. Fluids 35 (10), 11261136.

Arthur, D. \& VAssilvitskil, S. 2007 k-means++: The advantages of careful seeding. In Proc. of the 18th Annual ACM-SIAM Symposium on Discrete Algorithms, pp. 1027-1035. Philadelphia, PA, USA: Society for Industrial and Applied Mathematics.

Bansal, M. S. \& Yarusevych, S. 2017 Experimental study of flow through a cluster of three equally spaced cylinders. Exp. Thermal Fluid Sci. 80, 203-217.

Barros, D., Borée, J., Noack, B. R., Spohn, A. \& Ruiz, T. 2016 Bluff body drag manipulation using pulsed jets and Coanda effect. J. Fluid Mech. 805, 442-459.

BEARMAN, PW 1967 The effect of base bleed on the flow behind a two-dimensional model with a blunt trailing edge. Aeronautical Quarterly 18 (03), 207-224.

Berkooz, G., Holmes, P. \& Lumley, J. L. 1993 The proper orthogonal decomposition in the analysis of turbulent flows. Ann. Rev. Fluid Mech. 25, 539-575.

Boris, J. P., Grinstein, F. F., Oran, E. S. \& Kolbe, R. L. 1992 New insights into large eddy simulation. Fluid Dyn. Res. 10 (4-6), 199-228.

Brunton, S. L. \& NoAck, B. R. 2015 Closed-loop turbulence control: Progress and challenges. Appl. Mech. Rev. 67 (5), 050801:01-48.

Burkardt, J., Gunzburger, M. \& Lee, H. C. 2006 POD and CVT-based reduced-order modeling of Navier-Stokes flows. Comput. Methods Appl. Mech. Eng. 196, 337-355.

Cox, T. F. \& Cox, M. A. A. 2000 Multidimensional Scaling, 2nd edn., Monographs on Statistics and Applied Probability, vol. 88. Chapman and Hall.

Du, Y., Symeonidis, V. \& Karniadakis, G. E. 2002 Drag reduction in wall-bounded turbulence via a transverse travelling wave. J. Fluid Mech. 457, 1-34.

Duriez, T., Brunton, S. L. \& NoAck, B. R. 2016 Machine Learning Control - Taming Nonlinear Dynamics and Turbulence, Fluid Mechanics and Its Applications, vol. 116. Springer-Verlag.

Endres, D. M. \& Schindelin, J. E. 2003 A new metric for probability distributions. IEEE Transactions on Information Theory 49, 1858-1860.

García-Mayoral, Ricardo \& JimÉnez, Javier 2011 Hydrodynamic stability and breakdown of the viscous regime over riblets. J. Fluid Mech. 678, 317-347.

Geropp, D. 1995 Process and device for reducing the drag in the rear region of a vehicle, for example, a road or rail vehicle or the like. United States Patent US 5407245 A.

Geropp, D. \& Odenthal, H.-J. 2000 Drag reduction of motor vehicles by active flow control using the Coanda effect. Exp. Fluids 28 (1), 74-85.

Haller, G. 2005 An objective definition of a vortex. J. Fluid Mech. 525, 1-26.

Hirt, CW, Amsden, AA \& CoOK, JL 1997 An arbitrary Lagrangian-Eulerian computing method for all flow speeds. J. Comput. Phys. 135 (2), 203-216.

Hu, J. \& Zhou, Y. 2008a Flow structure behind two staggered circular cylinders. Part 1. Downstream evolution and classification. J. Fluid Mech. 607, 51-80.

Hu, J. \& Zhou, Y. $2008 b$ Flow structure behind two staggered circular cylinders. Part 2: Heat and momentum transport. J. Fluid Mech.607, 81-107.

Iтон, M., Tamano, S., Yokota, K. \& Taniguchi, S. 2006 Drag reduction in a turbulent boundary layer on a flexible sheet undergoing a spanwise traveling wave motion. J. Turbul. 7, N27.

Jeong, J. \& Hussain, F. 1995 On the identification of a vortex. J. Fluid Mech. 285, 69-94.

Jung, W. J., Mangiavacchi, N. \& Akhavan, R. 1992 Suppression of turbulence in wallbounded flows by highfrequency spanwise oscillations. Phys. Fluids A 4 (8), 16051607.

Kaiser, E., Li, R. \& NoAck, B. R. $2017 a$ On the control landscape topology. In The 20th 
World Congress of the International Federation of Automatic Control (IFAC), pp. 1-4. Toulouse, France.

Kaiser, E., Noack, B. R., Cordier, L., Spohn, A., Segond, M., Abel, M. W., Daviller, G., Östh, J., Krajnović, S. \& Niven, R. K. 2014 Cluster-based reduced-order modelling of a mixing layer. J. Fluid Mech. 754, 365-414.

Kaiser, E., Noack, B. R., Spohn, A., Cattafesta, L. N. \& Morzyński, M. $2017 b$ Clusterbased control of a separating flow over a smoothly contoured ramp. Theor. Comput. Fluid Dyn. 31, 579-593.

Kasten, J., Reininghaus, J., Hotz, I., Hege, H.-C., Noack, B. R., Daviller, G., Comte, P. \& Morzyński, M. 2016 Acceleration feature points of unsteady shear flows. Arch. Mech. 68, 55-80.

Klumpr, S., Meinke, M. \& Schröder, W. 2010a Numerical simulation of riblet controlled spatial transition in a zero-pressure-gradient boundary layer. Flow Turbul. Combust. 85 (1), 57-71.

Klumpr, S., Meinke, M. \& SchröDer, W. 2010b Drag reduction by spanwise transversal surface waves. J. Turbul. 11, N22.

Klumpr, S., Meinke, M. \& SchröDer, W. 2011 Friction drag variation via spanwise transversal surface waves. Flow Turbul. Combust. 87 (1), 33-53.

Koh, S.R., Meysonnat, P., Statnikov, V., Meinke, M. \& Schröder, W. $2015 a$ Dependence of turbulent wall-shear stress on the amplitude of spanwise transversal surface waves. Comput. Fluids 119, 261-275.

Koh, Seong Ryong, Meysonnat, Pascal, Meinke, Matthias \& Schröder, W. $2015 b$ Drag reduction via spanwise transversal surface waves at high reynolds numbers. Flow Turbul. Combust. 95 (1), 169-190.

KullbaCK, S. 1959 Information Theory and Statistics, 1st edn. New York: John Wiley.

Kullback, S. \& Leibler, R. A. 1951 On information and sufficiency. Annals Math. Stat. 22, $79-86$.

Li, W., Jessen, W., Roggenkamp, D., Klaas, M., Silex, W., Schiek, M. \& Schröder, W. 2015 Turbulent drag reduction by spanwise traveling ribbed surface waves. Eur. J. Mech. B. Fluids 53, 101-112.

Liou, M.-S. \& Steffen, C.J. 1993 A new flux splitting scheme. J. Comput. Phys. 107, 23-39.

Loiseau, J.-Ch., Noack, B. R. \& Brunton, S. L. 2018 Sparse reduced-order modeling: Sensor-based dynamics to full-state estimation. J. Fluid Mech. 844, 459-490.

LOYD, S. 1982 Least squares quantization in pcm. IEEE Trans. Inform. Theory 28 (2), 129-137.

Lugt, H.J. 1996 Introduction to Vortex Theory. Potomac (Maryland, U.S.A.): Vortex Flow Press.

MacQueEn, J. 1967 Some methods for classification and analysis of multivariate observations. Proc. of the Fifth Berkeley Symp. On Math. Stat. and Prob. 1, 281-297.

Meinke, M., Schröder, W., Krause, E. \& Rister, Th. 2002 A comparison of second-and sixth-order methods for large-eddy simulations. Comput. Fluids 31 (4), 695-718.

Meysonnat, P. S., Roggenkamp, D., Li, W., Roidl, B. \& Schröder, W. 2016 Experimental and numerical investigation of transversal traveling surface waves for drag reduction. Eur. J. Mech. B. Fluids 55, 313-323.

NoACK, B. R. 2016 From snapshots to modal expansions - bridging low residuals and pure frequencies. J. Fluid Mech. - Focus in Fluids 802, 1-4.

Noack, B. R., Afanasiev, K., Morzyński, M., Tadmor, G. \& Thiele, F. 2003 A hierarchy of low-dimensional models for the transient and post-transient cylinder wake. J. Fluid Mech. 497, 335-363.

NoAck, B. R. \& Morzyński, M. 2017 The fluidic pinball - a toolkit for multipleinput multiple-output flow control (version 1.0). Tech. Rep. 02/2017. Chair of Virtual Engineering, Poznan University of Technology, Poland.

Noack, B. R., Stankiewicz, W., Morzyński, M. \& Schmid, P. J. 2016 Recursive dynamic mode decomposition of transient and post-transient wake flows. J. Fluid Mech. 809, 843872.

Oxlade, A. R., Morrison, J. F., Qubain, A. \& Rigas, G. 2015 High-frequency forcing of a turbulent axisymmetric wake. J. Fluid Mech. 770, 305-318. 
Pastoor, M., Henning, L., Noack, B. R., King, R. \& Tadmor, G. 2008 Feedback shear layer control for bluff body drag reduction. J. Fluid Mech. 608, 161-196.

QuAdrIo, M. 2011 Drag reduction in turbulent boundary layers by in-plane wall motion. Philos. Trans. R. Soc. London, Ser. A 369 (1940), 1428-1442.

Raibaudo, C., Zhong, P., Martinuzzi, R. J. \& Noack, B. R. 2017 Closed-loop control of a triangular bluff body using rotating cylinders. In The 20th World Congress of the International Federation of Automatic Control (IFAC), pp. 1-6. Toulouse, France.

Renze, P., SchröDer, W. \& Meinke, M. 2008 Large-eddy simulation of film cooling flows at density gradients. Int. J. Heat Fluid Flow 29 (1), 18-34.

Roidl, B., Meinke, M. \& Schröder, W. 2013 A reformulated synthetic turbulence generation method for a zonal RANS-LES method and its application to zero-pressure gradient boundary layers. Int. J. Heat Fluid Flow 44, 28-40.

Rolland, R. 2017 Fluidic pinball - a control study. Master's thesis, LIMSI and ENSAM, Paris, France.

Roussopoulos, K. 1993 Feedback control of vortex shedding at low Reynolds numbers. J. Fluid Mech. 248, 267-296.

Rowley, C. W., Mezić, I., Bagheri, S., Schlatter, P. \& Henningson, D.S. 2009 Spectral analysis of nonlinear flows. J. Fluid Mech. 645, 115-127.

Rütten, F., Schröder, W. \& Meinke, M. 2005 Large-eddy simulation of low frequency oscillations of the Dean vortices in turbulent pipe bend flows. Phys. Fluids 17 (3), 035107.

Schmid, P. J. 2010 Dynamic mode decomposition for numerical and experimental data. J. Fluid. Mech 656, 5-28.

Schuster, H.G. 1988 Deterministic Chaos, 2nd edn. Weinheim: VCH Verlagsgesellschaft mbH.

Statnikov, V., Meinke, M. \& SchröDer, W. 2017 Reduced-order analysis of buffet flow of space launchers. J. Fluid Mech. 815, 125.

Steinhaus, H. 1956 Sur la division des corps matériels en parties. Bull. Acad. Polon. Sci. 4 (12), 801-804.

Tamano, Shinji \& Iтон, Мотоyuki 2012 Drag reduction in turbulent boundary layers by spanwise traveling waves with wall deformation. J. Turbul. 13, N9.

Theofilis, V. 2011 Global linear instability. Ann. Rev. Fluid Mech. 43, 319-352.

Thiria, B., Goujon-Durand, S. \& Wesfreid, J. E. 2006 The wake of a cylinder performing rotary oscillations. J. Fluid Mech. 560, 123-147.

Wood, C. J. 1964 The effect of base bleed on a periodic wake. Journal of the Royal Aeronautical Society 68 (643), 477-482.

Zhaо, H., Wu, J.-Z. \& Luo, J.-S. 2004 Turbulent drag reduction by traveling wave of flexible wall. Fluid Dyn. Res. 34 (3), 175-198. 